\title{
Congruence topologies on the mapping class group
}

\author{
Marco Boggi
}

July 12, 2021

\begin{abstract}
Let $\Gamma(S)$ be the pure mapping class group of a connected orientable surface $S$ of negative Euler characteristic. For $\mathscr{C}$ a class of finite groups, let $\hat{\pi}_{1}(S)^{\mathscr{C}}$ be the pro- $\mathscr{C}$ completion of the fundamental group of $S$. The $\mathscr{C}$-congruence completion $\check{\Gamma}(S)^{\mathscr{C}}$ of $\Gamma(S)$ is the profinite completion induced by the embedding $\Gamma(S) \hookrightarrow \operatorname{Out}\left(\hat{\pi}_{1}(S)^{\mathscr{C}}\right)$. In this paper, we begin a systematic study of such completions for different $\mathscr{C}$. We show that the combinatorial structure of the profinite groups $\check{\Gamma}(S)^{\mathscr{C}}$ closely resemble that of $\Gamma(S)$. A fundamental question is how $\mathscr{C}$-congruence completions compare with pro- $\mathscr{C}$ completions. Even though, in general (e.g. for $\mathscr{C}$ the class of finite solvable groups), $\check{\Gamma}(S)^{\mathscr{C}}$ is not even virtually a pro- $\mathscr{C}$ group, we show that, for $\mathbb{Z} / 2 \in \mathscr{C}$, $g(S) \leq 2$ and $S$ open, there is a natural epimorphism from the $\mathscr{C}$-congruence completion $\check{\Gamma}(S)(2)^{\mathscr{C}}$ of the abelian level of order 2 to its pro- $\mathscr{C}$ completion $\widehat{\Gamma}(S)(2)^{\mathscr{C}}$. In particular, this is an isomorphism for the class of finite groups and for the class of 2 -groups. Moreover, in these two cases, the result also holds for a closed surface.
\end{abstract}

MSC2010: 30F60, 14H10, 14H15, 32G15, 11F80, 14H30, 14F35.

\section{Introduction}

Let $S$ be a connected surface of negative Euler characteristic. We denote by $\Gamma(S)$ its pure mapping class group, that it is to say the group of isotopy classes of orientation preserving homeomorphism (or diffeomorphism) of $S$ in itself which do not permute punctures or boundary components of $S$.

For $S_{g}$ a closed orientable Riemann surface of genus $g$ and $\left\{P_{1}, \ldots, P_{n}\right\}$ a set of distinct points on $S_{g}$ such that $2 g-2+n>0$, we then let $S_{g, n}:=S_{g} \backslash\left\{P_{1}, \ldots, P_{n}\right\}$ and denote $\Gamma\left(S_{g, n}\right)$ by $\Gamma_{g, n}$. Let $\Pi_{g, n}:=\pi_{1}\left(S_{g, n}, P_{n+1}\right)$ be the fundamental group of $S_{g, n}$ based at $P_{n+1}$. There is a faithful representation:

$$
\rho_{g, n}: \Gamma_{g, n} \hookrightarrow \operatorname{Out}\left(\Pi_{g, n}\right) .
$$

A level of $\Gamma_{g, n}$ is just a finite index subgroup $H<\Gamma_{g, n}$. A characteristic finite index subgroup $K$ of $\Pi_{g, n}$ determines the geometric level $\Gamma_{K}$, defined to be the kernel of the induced representation:

$$
\rho_{K}: \Gamma_{g, n} \rightarrow \operatorname{Out}\left(\Pi_{g, n} / K\right)
$$


The abelian levels $\Gamma(m)$ of order $m$, for $m \geq 2$, are a particular case and are defined by the kernel of the natural representation:

$$
\rho_{(m)}: \Gamma_{g, n} \rightarrow \operatorname{Sp}\left(H_{1}\left(S_{g}, \mathbb{Z} / m\right)\right) .
$$

Definition 1.1. A class of finite groups is a full subcategory $\mathscr{C}$ of the category of finite groups which is closed under taking subgroups, homomorphic images and extensions (meaning that a short exact sequence of finite groups is in $\mathscr{C}$ whenever its exterior terms are). We always assume that $\mathscr{C}$ contains a nontrivial group. We denote by $\mathscr{F}$ the class of all finite groups, by $\mathscr{S}$ the class of finite solvable groups and by $(p)$, for $p>1$ a prime number, the class of $p$-groups.

We say that a subgroup $H$ of a group $G$ is $\mathscr{C}$-open if it contains a normal subgroup $N$ of $G$ such that the quotient group $G / N$ belongs to $\mathscr{C}$. In this case, we write $H \leq_{\mathscr{C}} G$ and $N \triangleleft_{\mathscr{C}} G$, respectively. For a finitely generated group $G$, a fundamental system of neighborhoods of the identity for the pro- $\mathscr{C}$ topology is given by any cofinal system of $\mathscr{C}$ open characteristic subgroups. The pro- $\mathscr{C}$ completion $\widehat{G}^{\mathscr{C}}$ is the completion of the group $G$ with respect to its pro- $\mathscr{C}$ topology. We denote $\widehat{G}^{\mathscr{F}}$ simply by $\widehat{G}$.

The pro- $\mathscr{C}$ topology on the fundamental group $\Pi_{g, n}$ determines a profinite topology on the mapping class group $\Gamma_{g, n}$ by associating to the set of characteristic $\mathscr{C}$-open subgroups of $\Pi_{g, n}$ the levels $\left\{\Gamma_{K}\right\}_{K \triangleleft_{\mathscr{C}} \Pi_{g, n}}$, which form a fundamental system of neighborhoods of the identity for a profinite topology on the mapping class group $\Gamma_{g, n}$.

We say that a subgroup of $\Gamma_{g, n}$ is $\mathscr{C}$-congruence open if it contains some level $\Gamma_{K}$ for $K \triangleleft_{\mathscr{C}} \Pi_{g, n}$. We call the topology thus defined the $\mathscr{C}$-congruence topology on $\Gamma_{g, n}$ and denote by $\check{\Gamma}_{g, n}^{\mathscr{C}}$ the corresponding completion. Also, for any subgroup $H$ of $\Gamma_{g, n}$, the $\mathscr{C}$-congruence topology on $H$ is the profinite topology whose fundamental system of neighborhoods of the identity is the set of normal finite index subgroups $\left\{H \cap \Gamma_{K}\right\}_{K \triangleleft_{\mathscr{C}} \Pi_{g, n}}$. The $\mathscr{C}$-congruence completion $\check{H}^{\mathscr{C}}$ of $H$ is the associated profinite completion.

The classical congruence subgroup problem asks whether the class of all finite groups of $\Pi_{g, n}$ induces on $\Gamma_{g, n}$ the full profinite topology, or, equivalently, if the set of geometric levels is cofinal in the set of finite index subgroups of $\Gamma_{g, n}$, ordered by inclusion. This problem is settled, affirmatively, only for $g \leq 2$ (cf. [4] and (ii) of Theorem 1.4).

Clearly, it is an interesting problem to understand the topology of $\breve{\Gamma}_{g, n}^{\mathscr{C}}$ for more general classes of finite groups. By Lemma 4.5.5 in [23], the $p$-congruence completion $\check{\Gamma}_{g, n}^{(p)}$ of $\Gamma_{g, n}$ is virtually a pro- $p$ group, that is to say, it contains an open subgroup which is pro- $p$. So it makes sense to ask whether $\check{\Gamma}_{g, n}^{(p)}$ is virtually the pro- $p$ completion of $\Gamma_{g, n}$. However, in general, it is not true that the profinite group $\check{\Gamma}_{g, n}^{\mathscr{C}}$ contains an open subgroup which is a pro- $\mathscr{C}$ group. In fact, in Proposition 2.5, we will show that, for $\mathscr{C}=\mathscr{S}$, the class of finite solvable groups, $\check{\Gamma}_{g, n}^{\mathscr{S}}$ is not virtually pro- $\mathscr{S}$. This motivates the following definition:

Definition 1.2. Let $\mathscr{C}$ be a class of finite groups:

(i) The mapping class group $\Gamma_{g, n}$ satisfies the $\mathscr{C}$-congruence subgroup property if $\check{\Gamma}_{g, n}^{\mathscr{C}}$ is virtually the pro- $\mathscr{C}$ completion of $\Gamma_{g, n}$, that is to say, for some $\mathscr{C}$-congruence open subgroup $U$ of $\Gamma_{g, n}$, denoting by $\check{U}^{\mathscr{C}}$ its $\mathscr{C}$-congruence completion, we have $\check{U}^{\mathscr{C}} \equiv \hat{U}^{\mathscr{C}}$. 
(ii) The mapping class group $\Gamma_{g, n}$ satisfies the weak $\mathscr{C}$-congruence subgroup property if there is a $\mathscr{C}$-congruence open subgroup $U$ of $\Gamma_{g, n}$ which is residually a $\mathscr{C}$-group and such that the $\mathscr{C}$-congruence topology induces on $U$ a topology which is finer than the pro- $\mathscr{C}$ topology. Equivalently: there is a natural epimorphism $\check{U}^{\mathscr{C}} \rightarrow \hat{U}^{\mathscr{C}}$.

Remark 1.3. In Definition 1.2 , (i) implies (ii). If $\check{\Gamma}_{g, n}^{\mathscr{C}}$ is virtually pro- $\mathscr{C}$, (i) and (ii) are equivalent. In (ii), we ask that $U$ is residually a $\mathscr{C}$-group in order to avoid that the property may hold trivially. For instance, if we let $U=\Gamma_{g, n}$, then the pro- $\mathscr{S}$ completion of $U$ is trivial for $g \geq 3$ (cf. Theorem 5.2 in [9]). Instead, for a prime $p \geq 0$, it is well known (cf. Section 2.1) that the abelian level $\Gamma(p)$ of $\Gamma_{g, n}$ is residually a $p$-group and so residually a $\mathscr{C}$-group for any class of finite groups $\mathscr{C}$ such that $\mathbb{Z} / p \in \mathscr{C}$.

In Corollary 5.4, we will show that the weak $\mathscr{C}$-congruence subgroup property is indeed satisfied in genus 0 for any class of finite groups $\mathscr{C}$. The next cases to consider then are genus 1 and 2, which are both subsumed under the notion of hyperelliptic mapping class group, which we define next.

For $g \geq 2$, let us fix a hyperelliptic involution $\iota$ in the mapping class group $\Gamma_{g}$. The hyperelliptic mapping class group $\Upsilon_{g}$ is the centralizer of $\iota$ in $\Gamma_{g}$. For $n>0$, we then define the hyperelliptic mapping class group $\Upsilon_{g, n}$ to be the inverse image of $\Upsilon_{g}$ via the natural epimorphism $\Gamma_{g, n} \rightarrow \Gamma_{g}$. Thus, for $g=2$, there holds $\Upsilon_{2, n}=\Gamma_{2, n}$. For $g=1$ and $n \geq 1$, we let instead $\Upsilon_{1, n}:=\Gamma_{1, n}$. From the definition, it follows that there is a natural faithful representation:

$$
\rho_{g, n}: \Upsilon_{g, n} \hookrightarrow \operatorname{Out}\left(\Pi_{g, n}\right) .
$$

The definition of the hyperelliptic mapping class group can be justified geometrically as follows. Let $\mathcal{M}_{g, n}$, for $2 g-2+n>0$, be the moduli stack of $n$-pointed, genus $g$, smooth algebraic complex curves. It is a smooth connected Deligne-Mumford stack (briefly $D-M$ stack) over $\mathbb{C}$ of dimension $3 g-3+n$, whose associated underlying complex analytic and topological étale groupoids, we both denote by $\mathcal{M}_{g, n}$ as well.

For stacks of the kind of $\mathcal{M}_{g, n}$, the fundamental group can be described in a simple way. In fact, $\mathcal{M}_{g, n}$ has a universal cover $T_{g, n}$ in the category of analytic manifolds. The fundamental group $\pi_{1}\left(\mathcal{M}_{g, n},[C]\right)$ is then identified with the deck transformation group of the universal cover $T_{g, n} \rightarrow \mathcal{M}_{g, n}$.

The choice of a lift of a point $[C] \in \mathcal{M}_{g, n}$ to $T_{g, n}$ and of a diffeomorphism $S_{g, n} \rightarrow$ $C \backslash\{$ marked points $\}$ identifies the mapping class group $\Gamma_{g, n}$ with $\pi_{1}\left(\mathcal{M}_{g, n},[C]\right)$. The representation:

$$
\rho_{g, n}: \pi_{1}\left(\mathcal{M}_{g, n},[C]\right) \rightarrow \operatorname{Out}\left(\Pi_{g, n}\right),
$$

induced by the identification of $\Gamma_{g, n}$ with $\pi_{1}\left(\mathcal{M}_{g, n},[C]\right)$, is equivalent to the universal monodromy representation associated to the short exact sequence of topological fundamental groups, determined by the $n$-punctured, genus $g$, universal curve $\mathcal{C}_{g, n} \rightarrow \mathcal{M}_{g, n}$.

Let us assume that the point $[C] \in \mathcal{M}_{g, n}$ parameterizes a hyperelliptic curve $C$ carrying the hyperelliptic involution $\iota$. The hyperelliptic mapping class group $\Upsilon_{g, n}$ then identifies with the fundamental group based at $[C]$ of the substack $\mathcal{H}_{g, n}$ of $\mathcal{M}_{g, n}$ which parameterizes 
hyperelliptic curves. Observe that, in genus 1 and 2, all smooth projective curves are hyperelliptic, i.e. admit a finite morphism of degree 2 onto $\mathbb{P}^{1}$.

As above, we define a level of $\Upsilon_{g, n}$ as a finite index subgroup $H<\Upsilon_{g, n}$. A characteristic finite index subgroup $K$ of $\Pi_{g, n}$ determines the geometric level $\Upsilon_{K}:=\Upsilon_{g, n} \cap \Gamma_{K}$, also defined as the kernel of the induced representation:

$$
\rho_{K}: \Upsilon_{g, n} \rightarrow \operatorname{Out}\left(\Pi_{g, n} / K\right)
$$

In this way, we also get the abelian level $\Upsilon(m):=\Upsilon_{g, n} \cap \Gamma(m)$ of order $m$, for $m \geq 2$.

The pro- $\mathscr{C}$ topology on the fundamental group $\Pi_{g, n}$ determines the $\mathscr{C}$-congruence topology on $\Upsilon_{g, n}$ with fundamental system of neighborhoods of the identity $\left\{\Upsilon_{K}\right\}_{K \triangleleft_{\mathscr{C}} \Pi_{g, n}}$. A subgroup of $\Upsilon_{g, n}$ is $\mathscr{C}$-congruence open if it contains some level $\Upsilon_{K}$ for $K \triangleleft_{\mathscr{C}} \Pi_{g, n}$. Let $\check{\Upsilon}_{g, n}^{\mathscr{C}}$ be the corresponding profinite completion of $\Upsilon_{g, n}$ which we call the $\mathscr{C}$-congruence

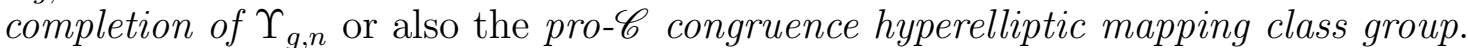

The kernel of the natural epimorphism $\Gamma_{g, n+1} \rightarrow \Gamma_{g, n}$, induced filling in the last puncture, identifies with the fundamental group $\Pi_{g, n}$. The $\mathscr{C}$-congruence completion $\check{\Pi}_{g, n}^{\mathscr{C}}$ of $\Pi_{g, n}$ is then the completion with respect to the profinite topology induced on $\Pi_{g, n}$ by the $\mathscr{C}$-congruence topology of $\Gamma_{g, n+1}$. We will see (cf. Proposition 2.4) that there is a natural epimorphism $\check{\Pi}_{g, n}^{\mathscr{C}} \rightarrow \widehat{\Pi}_{g, n}^{\mathscr{C}}$, which is an isomorphism for $\mathscr{C}=\mathscr{F}$ or $(p)$. In general, however, this map is not an isomorphism (e.g. for $\mathscr{C}=\mathscr{S}$ ) and it is a difficult and subtle question to determine when this happens. In Section 6, we will prove the following theorem:

Theorem 1.4. Let $\mathscr{C}$ be a class of finite groups such that $\mathbb{Z} / 2 \in \mathscr{C}$ and assume that $2 g-2+n>0$ and $g \geq 1$ :

(i) For $n \geq 1$, the weak $\mathscr{C}$-congruence property holds for $\Upsilon_{g, n}$. More precisely, there is a natural epimorphism of profinite groups $\check{\Upsilon}(2)^{\mathscr{C}} \rightarrow \widehat{\Upsilon}(2)^{\mathscr{C}}$.

(ii) If $\mathscr{C}$ is such that $\check{\Pi}_{g, k}^{\mathscr{C}} \equiv \widehat{\Pi}_{g, k}^{\mathscr{C}}$, for all $k \geq 0$ (e.g. $\mathscr{C}=\mathscr{F}$ or (2)), then the profinite group $\check{\Upsilon}_{g, n}^{\mathscr{C}}$ is virtually the pro- $\mathscr{C}$ completion of $\Upsilon_{g, n}$. More precisely, there is a natural isomorphism $\check{\Upsilon}(2)^{\mathscr{C}} \equiv \widehat{\Upsilon}(2)^{\mathscr{C}}$.

A few comments are in order. As a particular case of (ii) of Theorem 1.4, we obtain the subgroup congruence property for hyperelliptic mapping class groups in the closed surface case which was left open in [4].

For $\mathscr{C}$ the class of 2 -groups and $g=1$, (ii) of Theorem 1.4 has already been proved in [14] (for $\mathscr{C}=\mathscr{F}$ and $g=1$, this is instead a classical result by Asada [2]). In the same paper, the authors prove that the pro- $p$ congruence subgroup property fails for $\Gamma_{1,1}$ if $p \geq 11$. We expect that the same is true in genus $>1$, so that the hypothesis $\mathbb{Z} / 2 \in \mathscr{C}$ is probably not just a consequence of the proof. We instead believe that the hypothesis $n \geq 1$ in the first item of the theorem is a consequence of the proof.

We ignore whether the standard version of the subgroup congruence property holds for classes $\mathscr{C}$ of finite groups other than $\mathscr{F}$ or (2). Ultimately, this issue is related to the 
fact that the pro- $\mathscr{C}$ completion functor is right exact but, in general, not left exact (cf. Remark 4.11).

Even though the (weak) $\mathscr{C}$-congruence property for mapping class groups was the original motivation behind this paper, at a more technical level, its core lies in the study of the combinatorial properties of pro- $\mathscr{C}$ congruence mapping class groups developed in Section 3 and 4. The upshot is a description of centralizers of multitwist in the pro- $\mathscr{C}$ congruence mapping class groups. This then yields a description of their centers and the Birman exact sequences (3) and (9) which are central for the proof of Theorem 1.4. These generalize results originally obtained by Matsumoto, Hoshi and Mochizuki (cf. Remark 4.8).

In Section 7, we prove a generalized surface group version of a conjecture of Gelander and Lubotzky, proved for free groups of finite rank in [21]. Even though these results are essentially independent from the rest of the paper, they establish interesting connections with Grothendieck-Teichmüller theory and low-dimensional topology.

Acnowledgements. I thank Eduard Looijenga and Pavel Zalesskii for some useful conversations and suggestions.

\section{$2 \mathscr{C}$-congruence topologies on mapping class groups}

A $\mathscr{C}$-congruence topology on the mapping class group $\Gamma\left(S, x_{1}, \ldots, x_{n}\right)$, for $S$ a connected oriented surface of finite type and a given a set $\left\{x_{1}, \ldots, x_{n}\right\}$ of distinct points on $S$, is the topology that it acquires as an (outer) automorphism group of some fundamental group, which we can associate to the datum of the pointed surface $\left(S, x_{1}, \ldots, x_{n}\right)$, endowed with its $\mathscr{C}$-topology. As we will see below, there are several natural choices which lead to a priori distinct $\mathscr{C}$-congruence topologies. However, as we will see in Sections 4.4 and 4.10, for some of the most interesting cases (e.g. for $\mathscr{C}=\mathscr{F}$ or $(p)$ ) all of them determine the same topology on the mapping class group.

\subsection{The standard $\mathscr{C}$-congruence topologies}

For $x \in S$ a base point, let $\Pi:=\pi_{1}(S, x), S_{x}:=S \backslash\{x\}$ and $\Pi_{x}:=\pi_{1}\left(S_{x}, y\right)$, for $y \in S_{x}$. Then, Aut $\left(\widehat{\Pi}^{\mathscr{C}}\right)$ and Out $\left(\widehat{\Pi}^{\mathscr{C}}\right)$ are profinite groups (cf. Theorem 1.1 in [20]) and the natural representations $\Gamma(S) \rightarrow \operatorname{Out}\left(\Pi^{\mathscr{C}}\right)$ and $\Gamma\left(S_{x}\right) \cong \Gamma(S, x) \rightarrow \operatorname{Aut}\left(\Pi^{\mathscr{C}}\right)$ are faithful. In fact, since $\mathscr{C}$ contains $(p)$ for some prime $p$, it suffices to prove the claim for $\mathscr{C}=(p)$, which, in this case, follows from the $p$-separability and conjugacy $p$-separability of surface groups (cf. [22] or also Theorem A.1 and Theorem 4.3 in [7]).

Definition 2.1. The $\mathscr{C}$-congruence topology of $\Gamma(S)$ (resp. $\Gamma(S, x))$ is the topology inherited from the embedding $\Gamma(S) \hookrightarrow \operatorname{Out}\left(\widehat{\Pi}^{\mathscr{C}}\right)\left(\operatorname{resp} . \Gamma(S, x) \hookrightarrow \operatorname{Aut}\left(\widehat{\Pi}^{\mathscr{C}}\right)\right)$ and its $\mathscr{C}$ congruence completion, denoted $\check{\Gamma}(S)^{\mathscr{C}}$ (resp. $\left.\check{\Gamma}(S, x)^{\mathscr{C}}\right)$, is the associated completion or, equivalently, the closure of the image of this embedding. We also refer to these groups as to the pro- $\mathscr{C}$-congruence mapping class groups $\check{\Gamma}(S)^{\mathscr{C}}$ and $\check{\Gamma}(S, x)^{\mathscr{C}}$. 
A basic and very subtle question about the $\mathscr{C}$-congruence topologies introduced above is whether the natural isomorphism of mapping class groups $\Gamma\left(S_{x}\right) \cong \Gamma(S, x)$ induces an isomorphism $\check{\Gamma}\left(S_{x}\right)^{\mathscr{C}} \cong \check{\Gamma}(S, x)^{\mathscr{C}}$ of the respective $\mathscr{C}$-congruence completions. A related issue, which will turn out to be actually equivalent, is whether the two $\mathscr{C}$-congruence topologies induce the same topology on the fundamental group $\Pi$ considered as a subgroup of $\Gamma\left(S_{x}\right)$ and $\Gamma(S, x)$ respectively.

Since the center of a pro- $\mathscr{C}$ surface group is trivial (cf. Lemma 2.3), the natural homomorphism inn: $\Pi \rightarrow \Gamma(S, x) \subset \operatorname{Aut}\left(\widehat{\Pi}^{\mathscr{C}}\right)$ induces on $\Pi$ the pro- $\mathscr{C}$ topology and the natural short exact sequence $1 \rightarrow \widehat{\Pi}^{\mathscr{C}} \rightarrow \operatorname{Aut}\left(\widehat{\Pi}^{\mathscr{C}}\right) \rightarrow \operatorname{Out}\left(\widehat{\Pi}^{\mathscr{C}}\right) \rightarrow 1$ determines a Birman short exact sequence of profinite groups:

$$
1 \rightarrow \widehat{\Pi}^{\mathscr{C}} \rightarrow \check{\Gamma}(S, x)^{\mathscr{C}} \rightarrow \check{\Gamma}(S)^{\mathscr{C}} \rightarrow 1
$$

However, it is not clear whether the series of homomorphisms $\Pi \rightarrow \Gamma\left(S_{x}\right) \subset \operatorname{Out}\left(\widehat{\Pi}_{x}^{\mathscr{C}}\right)$ induces the pro- $\mathscr{C}$ topology on $\Pi$. In this case, an element $\gamma \in \Pi$ whose free homotopy class contains a simple closed curve is sent to the bounding pair map on $S_{x}$ associated to a tubular neighborhood of a simple representative of $\gamma$. We call the topology induced by the associated embedding $\Pi \subset \operatorname{Out}\left(\widehat{\Pi}_{x}^{\mathscr{C}}\right)$ the $\mathscr{C}$-congruence topology on $\Pi$ and denote by $\check{\Pi}^{\mathscr{C}}$ the corresponding profinite completion. In Proposition 2.4, we will show that, for $\mathscr{C}=\mathscr{F}$ or $(p)$, we have $\check{\Pi}^{\mathscr{C}} \equiv \widehat{\Pi}^{\mathscr{C}}$ and, in Corollary 4.5 , we will show that $\check{\Gamma}\left(S_{x}\right)^{\mathscr{C}} \cong \check{\Gamma}(S, x)^{\mathscr{C}}$, if and only if, $\check{\Pi}^{\mathscr{C}} \equiv \widehat{\Pi}^{\mathscr{C}}$.

\subsection{The tangential base point representation}

From the natural isomorphism of mapping class groups $\Gamma\left(S_{x}\right) \cong \Gamma(S, x)$, we deduce that there is a (faithful) representation $\Gamma\left(S_{x}\right) \rightarrow \operatorname{Aut}\left(\widehat{\Pi}^{\mathscr{C}}\right)$. However, it is not clear whether this representation factors through the natural embedding $\Gamma\left(S_{x}\right) \subset \check{\Gamma}\left(S_{x}\right)^{\mathscr{C}}$ and then a representation $\check{\Gamma}\left(S_{x}\right)^{\mathscr{C}} \rightarrow \operatorname{Aut}\left(\widehat{\Pi}^{\mathscr{C}}\right)$ with image $\check{\Gamma}(S, x)^{\mathscr{C}}$. In this section, we will show that this is the case.

Let us fix a tangential base point at $x \in S$ for the surface $S_{x}=S \backslash\{x\}$. To be precise, a base point $x \in S$ and a ray in the tangent space $T_{x} S$. Let $q: S_{\circ} \rightarrow S$ be the real oriented blow-up of $S$ at $x$. Then, $S_{\circ}$ is a surface with boundary whose interior is mapped diffeomorphically onto $S_{x}$ and whose boundary $\partial S_{\circ}$ is the preimage of $x$. The points of $\partial S_{\circ}$ are in bijective correspondence with the rays in the tangent space $T_{x} S$ so that the given ray defines a base point $x_{\circ} \in \partial S_{\circ}$. We put $\Pi_{\circ}:=\pi_{1}\left(S_{\circ}, x_{\circ}\right)$ so that $\Gamma\left(S_{\circ}\right)$ is a subgroup of the outer automorphism group of $\Pi_{\circ}$ and we let $\check{\Gamma}\left(S_{\circ}\right)^{\mathscr{C}}$ to be the profinite completion induced by the monomorphism $\Gamma\left(S_{\circ}\right) \hookrightarrow \operatorname{Out}\left(\widehat{\Pi}_{\circ}^{\mathscr{C}}\right)$. The natural isomorphism $\Gamma\left(S_{\circ}\right) \cong \Gamma\left(S_{x}\right)$ then induces an isomorphism of the respective $\mathscr{C}$-congruence completions $\check{\Gamma}\left(S_{\circ}\right)^{\mathscr{C}} \cong \check{\Gamma}\left(S_{x}\right)^{\mathscr{C}}$.

Let $u \in \Pi_{\circ}$ be an element whose free isotopy class contains $\partial S_{\circ}$, let $\mathscr{U}:=\{u\}^{\widehat{\Pi}_{\circ}^{\mathscr{C}}}$ be its conjugacy class in $\widehat{\Pi}_{\circ}^{\mathscr{C}}$, let then $\operatorname{Aut}_{u}\left(\widehat{\Pi}_{\circ}^{\mathscr{C}}\right)$ and Out $\mathscr{U}\left(\widehat{\Pi}_{\circ}^{\mathscr{C}}\right)$ be the stabilizers of $u$ and $\mathscr{U}$ in the groups Aut $\left(\widehat{\Pi}_{\circ}^{\mathscr{C}}\right)$ and $\operatorname{Out}\left(\widehat{\Pi}_{\circ}^{\mathscr{C}}\right)$, respectively. Observe that inn $u$ lies in the center of the group $\operatorname{Aut}_{u}\left(\widehat{\Pi}_{\circ}^{\mathscr{C}}\right)$. 
Lemma 2.2. Let us denote by $\operatorname{Inn}\left(u^{\hat{\mathbb{Z}}^{\mathscr{C}}}\right)$ the procyclic subgroup of $\operatorname{Aut}\left(\widehat{\Pi}_{\mathrm{O}}^{\mathscr{C}}\right)$ generated by inn $u$. Then the natural homomorphism $\hat{\Theta}_{u}^{\mathscr{C}}: \operatorname{Aut}_{u}\left(\widehat{\Pi}_{\circ}^{\mathscr{C}}\right) / \operatorname{Inn}\left(u^{\hat{\mathbb{Z}}^{\mathscr{C}}}\right) \rightarrow \operatorname{Out}_{\mathscr{U}}\left(\widehat{\Pi}_{\circ}^{\mathscr{C}}\right)$ is an isomorphism of profinite groups.

Proof. Let us construct an inverse $\hat{\mathrm{K}}_{u}^{\mathscr{C}}$ for the homomorphism $\hat{\Theta}_{u}^{\mathscr{C}}$. For this, we need the following lemma from [8] (cf. Proposition 3.5 ibid.):

Lemma 2.3. Let $\mathscr{C}$ be an extension closed class of finite groups. Let $\widehat{\Pi}^{\mathscr{C}}$ be the pro- $\mathscr{C}$ completion of a non-abelian surface group $\Pi$. Let $x \neq 1$ be a primitive element of $\Pi$ considered as an element of $\widehat{\Pi}^{\mathscr{C}}$. Then, for all $n \in \mathbb{Z} \backslash\{0\}$, there holds:

$$
N_{\widehat{\Pi}_{\mathscr{C}}}\left(x^{n \hat{\mathbb{Z}}^{\mathscr{C}}}\right)=N_{\widehat{\Pi}^{\mathscr{C}}}\left(x^{\hat{\mathbb{Z}}^{\mathscr{C}}}\right)=x^{\hat{\mathbb{Z}}^{\mathscr{C}}} .
$$

From Lemma 2.3, it follows that for $f \in \operatorname{Out}_{\mathscr{U}}\left(\widehat{\Pi}_{\circ}^{\mathscr{C}}\right)$, modulo $\operatorname{Inn}\left(u^{\hat{\mathbb{Z}}^{\mathscr{C}}}\right)$, there is a unique lift $\tilde{f} \in \operatorname{Aut}_{u}\left(\widehat{\Pi}_{\circ}^{\mathscr{C}}\right)$. We then let $\mathrm{K}_{u}(f):=[\tilde{f}]$, where $[\tilde{f}]$ denotes the coset of $\tilde{f}$ in the quotient $\operatorname{Aut}_{u}\left(\widehat{\Pi}_{\circ}^{\mathscr{C}}\right) / \operatorname{Inn}\left(u^{\hat{\mathbb{Z}}^{\mathscr{C}}}\right)$. It is clear that $\mathrm{K}_{u} \circ \Theta_{u}=\mathrm{Id}$ and $\Theta_{u} \circ \mathrm{K}_{u}=\mathrm{Id}$, which completes the proof of Lemma 2.2.

The natural epimorphism $\widehat{\Pi}_{\circ}^{\mathscr{C}} \rightarrow \widehat{\Pi}^{\mathscr{C}}$ induces a natural continuous homomorphism

$$
\hat{\Omega}_{u}^{\mathscr{C}}: \operatorname{Aut}_{u}\left(\widehat{\Pi}_{\circ}^{\mathscr{C}}\right) / \operatorname{Inn}\left(u^{\hat{\mathbb{Z}}^{\mathscr{C}}}\right) \rightarrow \operatorname{Aut}\left(\widehat{\Pi}^{\mathscr{C}}\right) .
$$

Let us then observe that, even though the isomorphism $\hat{\mathrm{K}}_{u}^{\mathscr{C}}$ and the homomorphism $\hat{\Omega}_{u}^{\mathscr{C}}$ depend on the choice of $u \in \mathscr{U}$, their composition $\hat{\Omega}_{u}^{\mathscr{C}} \circ \hat{\mathrm{K}}_{u}^{\mathscr{C}}$ only depends on the conjugacy class $\mathscr{U}$, which is determined by the point $x \in S$. Therefore, restricting $\hat{\Omega}_{u}^{\mathscr{C}} \circ \hat{\mathrm{K}}_{u}^{\mathscr{C}}$ to $\check{\Gamma}\left(S_{x}\right)^{\mathscr{C}} \equiv \check{\Gamma}\left(S_{\circ}\right)^{\mathscr{C}}$, we obtain a natural continuous epimorphism

$$
\hat{\Delta}_{x}^{\mathscr{C}}: \check{\Gamma}\left(S_{x}\right)^{\mathscr{C}} \rightarrow \check{\Gamma}(S, x)^{\mathscr{C}},
$$

which only depends on the point $x \in S$. Hence, we obtain a natural representation $\check{\Gamma}\left(S_{x}\right)^{\mathscr{C}} \rightarrow \operatorname{Aut}\left(\widehat{\Pi}^{\mathscr{C}}\right)$ which extends the representation $\Gamma\left(S_{x}\right) \hookrightarrow \operatorname{Aut}\left(\widehat{\Pi}^{\mathscr{C}}\right)$ considered at the beginning of this section.

The kernel of $\hat{\Delta}_{x}^{\mathscr{C}}$ clearly measures the difference between the $\mathscr{C}$-congruence topologies on the mapping class group $\Gamma\left(S_{x}\right)$, associated to the representations $\Gamma\left(S_{x}\right) \hookrightarrow \operatorname{Out}\left(\hat{\pi}_{1}\left(S_{x}\right)^{\mathscr{C}}\right)$ and $\Gamma\left(S_{x}\right) \cong \Gamma(S, x) \hookrightarrow \operatorname{Aut}\left(\widehat{\Pi}^{\mathscr{C}}\right)$.

The epimorphism $\hat{\Delta}_{x}^{\mathscr{C}}$ can be recovered from the inner action of $\check{\Gamma}\left(S_{x}\right)^{\mathscr{C}}$ on the $\mathscr{C}$ congruence completion $\Pi^{\mathscr{C}}$ of $\Pi$. In Section 4.4 , we will actually show that $\operatorname{ker} \hat{\Delta}_{x}^{\mathscr{C}}=\{1\}$, if and only if, $\check{\Pi}^{\mathscr{C}} \equiv \widehat{\Pi}^{\mathscr{C}}$. For the moment, let us observe the following:

Proposition 2.4. Let $\check{\Pi}^{\mathscr{C}}$ be the $\mathscr{C}$-congruence completion of $\Pi$ defined in Section 2.1:

(i) There is a natural epimorphism $\check{\Pi}^{\mathscr{C}} \rightarrow \widehat{\Pi}^{\mathscr{C}}$.

(ii) For $\mathscr{C}=\mathscr{F}$ or $(p)$, we have $\check{\Pi}^{\mathscr{C}} \equiv \widehat{\Pi}^{\mathscr{C}}$.

Proof. The restriction to $\check{\Pi}^{\mathscr{C}}$ of the epimorphism $\hat{\Delta}_{x}^{\mathscr{C}}: \check{\Gamma}\left(S_{x}\right)^{\mathscr{C}} \rightarrow \check{\Gamma}(S, x)^{\mathscr{C}}$ induces an epimorphism $\check{\Pi}^{\mathscr{C}} \rightarrow \widehat{\Pi}^{\mathscr{C}}$, which is obviously an isomorphism for $\mathscr{C}=\mathscr{F}$. For $\mathscr{C}=(p)$, this instead follows from Lemma 4.5.5 in [23]. 


\subsection{The configuration space $\mathscr{C}$-congruence topology}

For a given a set $\left\{x_{1}, \ldots, x_{n}\right\}$ of distinct points on $S$ and $n \geq 2$, there is another natural representation to consider. Let us denote by $S(n)$ the configuration space of $n$ points on $S$, by $\bar{y}=:\left\{y_{1}, \ldots, y_{n}\right\}$ a point of $S(n)$ and by $\Pi(n)$ its fundamental group based at $\bar{x}=:\left\{x_{1}, \ldots, x_{n}\right\} \in S(n)$. Let $\Gamma(S, \bar{x})$ be the mapping class group of the $n$-pointed surface $\left(S, x_{1}, \ldots, x_{n}\right)$. A self-homeomorphism of the pointed surface $\left(S, x_{1}, \ldots, x_{n}\right)$ induces one of the pointed space $(S(n), \bar{x})$. There is then a natural faithful representation:

$$
\rho_{n}: \Gamma(S, \bar{x}) \hookrightarrow \operatorname{Aut}(\Pi(n))
$$

Alternatively, the representation $\rho_{n}$ is defined by restricting the inner automorphisms of $\Gamma(S, \bar{x})$ to its normal subgroup $\Pi(n)$. Let $\widehat{\Pi}(n)^{\mathscr{C}}$ be the pro- $\mathscr{C}$ completion of $\Pi(n)$, then $\rho_{n}$ induces a representation:

$$
\rho_{n}^{\mathscr{C}}: \Gamma(S, \bar{x}) \rightarrow \operatorname{Aut}\left(\widehat{\Pi}(n)^{\mathscr{C}}\right)
$$

We define the $\mathscr{C}$-congruence completion of $\Gamma(S, \bar{x})$ (or also the pro- $\mathscr{C}$-congruence mapping class group), denoted $\check{\Gamma}(S, \bar{x})^{\mathscr{C}}$, to be the closure of the image of the representation $\rho_{n}^{\mathscr{C}}$. In Section 4.5, we will compare this congruence topology with those introduced in Section 2.1.

\subsection{The $\mathscr{S}$-congruence topology is not virtually pro- $\mathscr{S}$}

In this section, we will show that, for $\mathscr{C}=\mathscr{S}$, the class of finite solvable groups, the pro- $\mathscr{S}$ congruence mapping class group $\check{\Gamma}_{g, n}^{\mathscr{S}}$ is not virtually pro- $\mathscr{S}$ :

Proposition 2.5. For $g \geq 1$, the pro-S $\mathscr{S}$ congruence mapping class group $\check{\Gamma}_{g, n}^{\mathscr{S}}$ is not

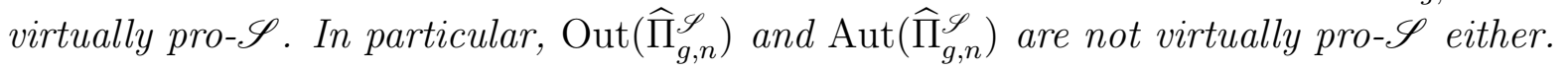

Proof. Let $\widehat{\Pi}_{g, n}^{\text {nil }}$ be the profinite nilpotent completion of $\Pi_{g, n}$. There is a natural epimorphism with characteristic kernel $\widehat{\Pi}_{g, n}^{\mathscr{S}} \rightarrow \widehat{\Pi}_{g, n}^{\text {nil }}$ and then a natural representation $\check{\Gamma}_{g, n}^{\mathscr{S}} \rightarrow$ $\operatorname{Out}\left(\widehat{\Pi}_{g, n}^{\text {nil }}\right)$. Let us denote by $\check{\Gamma}_{g, n}^{\text {nil }}$ the image of this representation. The decomposition $\operatorname{Aut}\left(\widehat{\Pi}_{g, n}^{\text {nil }}\right) \cong \prod_{p \text { prime }} \operatorname{Aut}\left(\widehat{\Pi}_{g, n}^{(p)}\right)$ induces one $\check{\Gamma}_{g, n}^{\text {nil }} \cong \prod_{p \text { prime }} \check{\Gamma}_{g, n}^{(p)}$. For every prime $p>0$, there is an epimorphism $\check{\Gamma}_{g, n}^{(p)} \rightarrow \operatorname{Sp}_{2 g}(\mathbb{Z} / p)$ and every open subgroup $U$ of $\check{\Gamma}_{g, n}^{\text {nil }}$ contains infinitely many factors $\check{\Gamma}_{g, n}^{(p)}$. Therefore, the group $\check{\Gamma}_{g, n}^{\text {nil }}$ is not virtually pro- $\mathscr{S}$ and so the same is true for $\check{\Gamma}_{g, n}^{\mathscr{S}}$.

Remark 2.6. By the methods of [2], it is not difficult to show that the abelian level $\check{\Gamma}_{1,1}^{\mathscr{S}}(4)$ identifies with an open subgroup of $\check{\Gamma}_{0,4}^{\mathscr{S}}$ (see also the short exact sequence (14)). Therefore, Proposition 2.5 also holds in genus 0 . We leave the details to the interested reader. 


\section{Modular subgroups of pro- $\mathscr{C}$-congruence mapping class groups}

A fundamental feature of the mapping class group $\Gamma(S)$ of a connected oriented surface of finite negative Euler characteristic $S$ is that the stabilizer of a set of isotopy classes of disjoint simple closed curves (embedded circles) on $S$ is described in terms of mapping class groups of the subsurfaces obtained cutting $S$ along those curves. In this section, we will show that a similar property holds for the pro- $\mathscr{C}$ congruence mapping class group.

\subsection{The closure of stabilizers}

The following theorem is a generalization to the pro- $\mathscr{C}$ case of Lemma 4.6 of [6]. Let us recall that a simple closed curve (an embedded circle) on $S$ is peripheral if it bounds on one side a disc with a single puncture. We denote by $\Gamma(S \backslash \gamma)$ the direct product of the pure mapping class groups of the connected components of the surface $S \backslash \gamma$ :

Theorem 3.1. Let $S$ be a connected oriented surface of finite negative Euler characteristic and let $\gamma \subset S$ be a non-peripheral simple closed curve on $S$. Denote by $\Gamma(S)_{\vec{\gamma}}$ the subgroup of elements of $\Gamma(S)$ which preserve the free homotopy class of $\gamma$ and a fixed orientation on it and by $\check{\Gamma}(S)_{\vec{\gamma}}^{\mathscr{C}}$ the closure of this subgroup in the $\mathscr{C}$-congruence completion $\check{\Gamma}(S)^{\mathscr{C}}$ of $\Gamma(S)$. Then there is a natural surjection $\check{\Gamma}(S)_{\vec{\gamma}}^{\mathscr{C}} \rightarrow \check{\Gamma}(S \backslash \gamma)^{\mathscr{C}}$ whose kernel is topologically generated by the Dehn twist $\tau_{\gamma}$ (making it isomorphic to $\hat{\mathbb{Z}}^{\mathscr{C}}$ ).

We will need the following lemma from [8] (cf. Theorem 3.6 ibid.):

Lemma 3.2. Let $\sigma$ be a closed one-dimensional submanifold of an oriented surface $S, S_{0}$ a connected component of $S \backslash \sigma$ and $x \in S_{0}$. Then the injection $\pi_{1}\left(S_{0}, x\right) \hookrightarrow \pi_{1}(S, x)$ induces an injection $\hat{\pi}_{1}\left(S_{0}, x\right)^{\mathscr{C}} \hookrightarrow \hat{\pi}_{1}(S, x)^{\mathscr{C}}$ with closed image. Moreover, that image is its own normalizer in $\hat{\pi}_{1}(S, x)^{\mathscr{C}}$.

Proof of Theorem 3.1. Every mapping class which fixes the homotopy class of $\gamma$ fixes its isotopy class and hence contains a member which leaves $\gamma$ invariant in an orientation preserving manner. It is easily checked that this identifies $\Gamma(S)_{\vec{\gamma}}$ with the connected component group of diffeomorphisms of $S$ having that property and so we have a natural homomorphism $\Gamma(S)_{\vec{\gamma}} \rightarrow \Gamma(S \backslash \gamma)$. This homomorphism is surjective, for every mapping class of $S \backslash \gamma$ is representable by a diffeomorphism that is the identity on the trace of a neighborhood of $\gamma$ on $S \backslash \gamma$. It is also easy to see that the kernel is generated by $\tau_{\gamma}$.

Denote by $q: S^{\gamma} \rightarrow S$ the real oriented blowup of $S$ along $\gamma$. This is a surface with boundary $\partial S^{\gamma}$. Its interior maps isomorphically onto $S \backslash \gamma$ and the preimage of $\gamma$ consists of two boundary components $\gamma_{ \pm}$, each mapping isomorphically onto $\gamma$ and corresponding to the choice of an orientation of $\gamma$. We choose a base point $x \in \gamma$ and denote by $x_{ \pm}$its preimage in $\gamma_{ \pm}$.

In what follows we assume that $S \backslash \gamma$ is connected, the disconnected case being somewhat easier to treat. We abbreviate $\Pi:=\pi_{1}(S, x)$ and $\Pi_{\gamma}:=\pi_{1}\left(S^{\gamma}, x_{-}\right)$. Then $\Pi$ can 
then be obtained from $\Pi_{\gamma}$ as follows: choose an embedded interval $\delta$ from $x_{-}$to $x_{+}$so that its image $q(\delta)$ in $S$ is an embedded circle and denote by $c_{-}, c_{+} \in \Pi_{\gamma}$ the elements defined by respectively $\gamma_{-}$and the loop which first traverses $\delta$, then $\gamma_{+}$and then returns via the inverse of $\delta$. Then $\Pi$ can be identified with the quotient of the free product of $\Pi_{\gamma}$ and a multiplicatively written copy $d^{\mathbb{Z}}$ of $\mathbb{Z}$ by the relation $d c_{-} d^{-1}=c_{+}$, with $d$ mapping to the class of $q(\delta)$ in $\Pi$. This is an instance of the HNN construction; in particular, the homomorphism $\Pi_{\gamma} \rightarrow \Pi$ is injective. According to Lemma 3.2, this induces an injection $\hat{\Pi}_{\gamma}^{\mathscr{C}} \rightarrow \hat{\Pi}^{\mathscr{C}}$ with closed image and with the property that this image is its own normalizer. We thus obtain a homomorphism $\Gamma(S)_{\vec{\gamma}} \rightarrow \operatorname{Out}\left(\Pi_{\gamma}\right)$ which enjoys the property that it is continuous for the $\mathscr{C}$ topology induced by $\Pi$ resp. $\Pi_{\gamma}$. This induces a homomorphism $\check{\Gamma}(S)_{\vec{\gamma}} \rightarrow \operatorname{Out}\left(\Pi_{\gamma}^{\mathscr{C}}\right)$ with image $\check{\Gamma}\left(S^{\gamma}\right) \cong \check{\Gamma}(S \backslash \gamma)$.

Now let $f$ be in the kernel of the surjection $\check{\Gamma}(S)_{\vec{\gamma}} \rightarrow \check{\Gamma}\left(S^{\gamma}\right)$. By definition $f$ lifts to an automorphism $\tilde{f}$ of $\hat{\Pi}^{\mathscr{C}}$ that is the identity on $\widehat{\Pi}_{\gamma}^{\mathscr{C}}$. In particular, $\tilde{f}$ fixes $c_{ \pm}$and so

$$
\tilde{f}(d) c_{-} \tilde{f}(d)^{-1}=\tilde{f}\left(d c_{-} d^{-1}\right)=\tilde{f}\left(c_{+}\right)=c_{+}=d c_{-} d^{-1} .
$$

Hence $d^{-1} \tilde{f}(d)$ centralizes $c_{-}$. By Proposition 3.5 in [8], this implies that $\tilde{f}(d)=d c_{-}^{k}$, for some $k \in \hat{\mathbb{Z}}^{\mathscr{C}}$. In terms of the HNN decomposition above, the profinite Dehn twist $\tau_{\gamma}^{-k}$ takes $d$ to $d c_{-}^{k}$ and induces the identity in $\widehat{\Pi}_{\gamma}^{\mathscr{C}}$. Since $\hat{\Pi}_{\gamma}^{\mathscr{C}}$ and $d$ generate a dense subgroup of $\hat{\Pi}^{\mathscr{C}}$, it follows that $f=\tau_{\gamma}^{-k}$.

\subsection{The complex of pro- $\mathscr{C}$ curves on $S$}

Sets of isotopy classes of disjoint simple closed curves on the surface $S$ can be arranged so that they form a simplicial complex:

Definition 3.3. A multicurve $\sigma$ on $S$ is a set of disjoint simple closed curves on $S$ such that $S \backslash \sigma$ does not contain discs or one-punctured discs. The complex of curves $C(S)$ is the abstract simplicial complex whose simplices are isotopy classes of multicurves on $S$.

For $S=S_{g, n}$, it is easy to check that the combinatorial dimension of $C\left(S_{g, n}\right)$ is $n-4$ for $g=0$ and $3 g-4+n$ for $g \geq 1$. There is a natural simplicial action of $\Gamma(S)$ on $C(S)$. In this subsection, we will define a pro- $\mathscr{C}$ version of the complex of curves $C(S)$, generalizing definitions and results given in Section 3 and 4 of [6].

Let $\mathscr{L}=C(S)_{0}$ be the set of isotopy classes of nonperipheral simple closed curves on $S$. Let $\Pi / \sim$ be the set of conjugacy classes of elements of $\Pi\left(:=\pi_{1}(S, x)\right)$ and let $\mathscr{P}_{2}(\Pi / \sim)$ be the set of unordered pairs of elements of $\Pi / \sim$. For a given $\gamma \in \Pi$, let us denote by $\gamma^{ \pm 1}$ the set $\left\{\gamma, \gamma^{-1}\right\}$ and by $\left[\gamma^{ \pm 1}\right]$ its equivalence class in $\mathscr{P}_{2}(\Pi / \sim)$. Let us then define the natural embedding $\iota: \mathscr{L} \hookrightarrow \mathscr{P}_{2}(\Pi / \sim)$, choosing, for an element $\gamma \in \mathscr{L}$, an element $\vec{\gamma}_{*} \in \Pi$ whose free homotopy class contains $\gamma$ and letting $\iota(\gamma):=\left[\vec{\gamma}_{*}^{ \pm 1}\right]$.

Let $\widehat{\Pi}^{\mathscr{C}} / \sim$ be the set of conjugacy classes of elements of $\widehat{\Pi}^{\mathscr{C}}$ and $\mathscr{P}_{2}\left(\widehat{\Pi}^{\mathscr{C}} / \sim\right)$ the profinite set of unordered pairs of elements of $\widehat{\Pi}^{\mathscr{C}} / \sim$. Since $\Pi$ is conjugacy $p$-separable (cf. [22] or Theorem $4.3[7]$ ), the set $\Pi / \sim$ embeds in the profinite set $\widehat{\Pi}^{\mathscr{C}} / \sim$. So, let 
us define the set of nonperipheral pro- $\mathscr{C}$ simple closed curves (or circles) $\hat{\mathscr{L}}^{\mathscr{C}}$ on $S$ to be the closure of the set $\iota(\mathscr{L})$ inside the profinite set $\mathscr{P}_{2}\left(\widehat{\Pi}^{\mathscr{C}} / \sim\right)$. An ordering of the set $\left\{\alpha, \alpha^{-1}\right\}$ is preserved by the conjugacy action and defines an orientation for the associated equivalence class $\left[\alpha^{ \pm 1}\right] \in \hat{\mathscr{L}}^{\mathscr{C}}$.

For all $k \geq 0$, there is a natural embedding of the set $C(S)_{k-1}$ of isotopy classes of multicurves on $S$ of cardinality $k$ into the profinite set $\mathscr{P}_{k}\left(\hat{\mathscr{L}}^{\mathscr{C}}\right)$ of unordered subsets of $k$ elements of $\hat{\mathscr{L}}^{\mathscr{C}}$. Let us then define the set of pro- $\mathscr{C}$ multicurves on $S$ as the union of the closures of the sets $C(S)_{k-1}$ inside the profinite sets $\mathscr{P}_{k}\left(\hat{\mathscr{L}}^{\mathscr{C}}\right)$, for all $k>0$.

A simplicial profinite complex is an abstract simplicial complex whose set of vertices is endowed with a profinite topology such that the sets of $k$-simplices, with the induced topologies, are compact and then profinite, for all $k \geq 0$. For these simplicial complexes, the procedure which associates to an abstract simplicial complex and an ordering of its vertex set a simplicial set produces a simplicial profinite set.

Definition 3.4. Let $L\left(\widehat{\Pi}^{\mathscr{C}}\right)$ be the abstract simplicial profinite complex whose simplices are pro- $\mathscr{C}$ multicurves on $S$. The abstract simplicial profinite complex $L\left(\widehat{\Pi}^{\mathscr{C}}\right)$ is called the complex of pro- $\mathscr{C}$ curves on $S$.

There is a natural continuous action of the pro- $\mathscr{C}$ congruence mapping class group $\check{\Gamma}(S)^{\mathscr{C}}$ on the complex of pro- $\mathscr{C}$ curves $L\left(\widehat{\Pi}^{\mathscr{C}}\right)$. There are finitely many orbits of $\check{\Gamma}(S)^{\mathscr{C}}$ in $L\left(\widehat{\Pi}^{\mathscr{C}}\right)_{k}$ each containing an element of $C(S)_{k}$, for $k \geq 0$. It is not difficult to show (cf. Section $4[6])$ that these orbits correspond to the possible topological types of the surface $S \backslash \sigma$, for $\sigma$ a multicurve on $S$.

Let $G$ be a discrete group acting with a finite number of orbits on a set $X$ and let $\tilde{G}$ be the profinite completion of $G$ with respect to an inverse system $\left\{U_{\lambda}\right\}_{\lambda \in \Lambda}$ of finite index normal subgroups. The $\tilde{G}$-completion $\tilde{X}$ of the set $X$ is defined to be the inverse limit $\lim _{\lambda \in \Lambda} X / U_{\lambda}$. The profinite set $\tilde{X}$ is naturally endowed with a continuous $\tilde{G}$-action and a $G$-equivariant map $X \rightarrow \tilde{X}$. It is characterized by the following universal property. Given a profinite set $Y$ endowed with a continuous $\tilde{G}$-action and a $G$-equivariant map $f: X \rightarrow Y$, there exists a unique continuous $\tilde{G}$-equivariant map $\tilde{f}: \tilde{X} \rightarrow Y$ extending $f$.

Let us also observe that, if $\tilde{U}$ is an open subgroup of $\tilde{G}$ and $U$ is its inverse image in $G$ via the canonical map $G \rightarrow \tilde{G}$, then a $G$-set $X$ is also a $U$-set and its $\tilde{U}$-completion is naturally isomorphic to its $\tilde{G}$-completion.

The main result of $\S 4$ in [6] (cf. Theorem 4.2 in [6]) is that, for $\mathscr{C}$ the class of all finite groups, the profinite set $\hat{\mathscr{L}}(S)$ is the $\check{\Gamma}(S)$-completion of the $\operatorname{discrete} \Gamma(S)$-set $\mathscr{L}(S)$, i.e. there is a natural continuous isomorphism of $\check{\Gamma}(S)$-sets:

$$
\hat{\mathscr{L}}(S) \cong \lim _{\longleftarrow}{ }_{\lambda \in \Lambda} \mathscr{L}(S) / \Gamma_{\lambda}
$$

where $\left\{\Gamma_{\lambda}\right\}_{\lambda \in \Lambda}$ is a tower of finite index normal subgroup of $\Gamma(S)$ which forms a fundamental system of neighbourhoods of the identity for the full congruence topology. In fact, this result holds for any extension closed class of finite groups $\mathscr{C}$ : 
Theorem 3.5. Let $\left\{\Gamma_{\lambda}\right\}_{\lambda \in \Lambda}$ be a tower of finite index normal subgroup of $\Gamma(S)$ which forms a fundamental system of neighbourhoods of the identity for the $\mathscr{C}$-congruence topology. There is then a natural continuous isomorphism of $\check{\Gamma}(S)^{\mathscr{C}}$-sets:

$$
\hat{\mathscr{L}}^{\mathscr{C}}(S) \cong \lim _{\varkappa}{ }_{\lambda \in \Lambda} \mathscr{L}(S) / \Gamma_{\lambda}
$$

Proof. In order to prove Theorem 3.5, we just need to rephrase the proof of Theorem 4.2 in [6] in terms of pro- $\mathscr{C}$ completions and $\mathscr{C}$-congruence completions instead of full profinite completions and full congruence completions of all the objects involved, where Lemma 4.3 in [6] is replaced by its pro- $\mathscr{C}$ version (cf. Lemma 2.3 in this paper).

Given a simplex $\sigma \in C(S)$, let us denote also by $\sigma$ its image in $L\left(\widehat{\Pi}^{\mathscr{C}}\right)$ and by $\check{\Gamma}(S)_{\sigma}^{\mathscr{C}}$ the corresponding $\check{\Gamma}(S)^{\mathscr{C}}$-stabilizer. From Proposition 6.5 in [3] and Theorem 3.5, it follows that $\check{\Gamma}(S)_{\sigma}^{\mathscr{C}}$ is the closure of the stabilizer $\Gamma(S)_{\sigma}$ (for the action of $\Gamma(S)$ on $C(S)$ ) in $\check{\Gamma}(S)^{\mathscr{C}}$.

Since every $\sigma \in L\left(\widehat{\Pi}^{\mathscr{C}}\right)$ is in the $\check{\Gamma}(S)^{\mathscr{C}}$-orbit of some simplex in the image of $C(S)$, this gives a complete description of stabilizers for the action of $\check{\Gamma}(S)^{\mathscr{C}}$ on the pro- $\mathscr{C}$ curve complex $L\left(\widehat{\Pi}^{\mathscr{C}}\right)$. The precise result is the following, where, for a simplex $\sigma \in C(S)$, we denote by $\Gamma(S \backslash \sigma)$ the direct product of the pure mapping class groups of the connected components of the surface $S \backslash \sigma$ :

Theorem 3.6. Let $\sigma \in L\left(\widehat{\Pi}^{\mathscr{C}}\right)$ be a simplex in the image of $C(S)$. Let $\Sigma_{\sigma^{ \pm}}$be the group of permutations on the set $\sigma^{ \pm}:=\sigma^{+} \cup \sigma^{-}$of oriented simple closed curves in $\sigma$. Then, the stabilizer $\check{\Gamma}(S)_{\sigma}^{\mathscr{C}}$ of $\sigma$ for the action of $\check{\Gamma}(S)^{\mathscr{C}}$ on $L\left(\widehat{\Pi}^{\mathscr{C}}\right)$ fits into the two exact sequences:

$$
\begin{gathered}
1 \rightarrow \check{\Gamma}(S)_{\vec{\sigma}}^{\mathscr{C}} \rightarrow \check{\Gamma}(S)_{\sigma}^{\mathscr{C}} \rightarrow \Sigma_{\sigma^{ \pm}}, \\
1 \rightarrow \prod_{i=0}^{k} \tau_{\gamma_{i}}^{\hat{\mathbb{Z}}^{\mathscr{C}}} \rightarrow \check{\Gamma}(S)_{\vec{\sigma}}^{\mathscr{C}} \rightarrow \check{\Gamma}(S \backslash \sigma)^{\mathscr{C}} \rightarrow 1 .
\end{gathered}
$$

Proof. Exactness of the upper sequence is obvious. Right exactness and exactness in the middle of the one below both follow from Theorem 3.1 and induction. Left exactness is equivalent to the claim that the levels $\Gamma^{K_{\ell},(m)}$ of $\Gamma(S)$ introduced in [5] induce the pro- $\mathscr{C}$ topology on the abelian subgroup $\prod_{i=0}^{k} \tau_{\gamma_{i}}^{\mathbb{Z}}$ of $\Gamma(S)$. The claim then follows from the explicit description of the local monodromy representation for these level structures given in Proposition 3.11 [5], where for $K$ we take a $\mathscr{C}$-open subgroup of $\Pi$ satisfying the hypotheses of Lemma $3.10[5]$ and for $\ell, m \geq 2$ integers such that $\mathbb{Z} / \ell$ and $\mathbb{Z} / m \in \mathscr{C}$.

\section{Centralizers of pro- $\mathscr{C}$ Dehn twists and centers of pro- $\mathscr{C}$ congruence mapping class groups}

The stabilizer in the mapping class group $\Gamma(S)$ of a set of isotopy classes of disjoint simple closed curves on $S$ is the centralizer of the product of the Dehn twists associated to those curves. We will show that this result holds also for the pro- $\mathscr{C}$ congruence mapping class group $\check{\Gamma}(S)^{\mathscr{C}}$ but the proof is much more difficult and requires to develop entirely different tools. This will allow to determine the centers of pro- $\mathscr{C}$ congruence mapping class groups and then to provide a Birman exact sequence for them. 


\subsection{A linearization of the complex of pro- $\mathscr{C}$ curves}

In this section, we will extend the results of Section 5 in [8] to the pro- $\mathscr{C}$ case. Let $K$ be an open characteristic subgroup of $\widehat{\Pi}^{\mathscr{C}}$ and let $p_{K}: S_{K} \rightarrow S$ be the associated normal unramified covering of Riemann surfaces with covering transformation group $G_{K}:=\widehat{\Pi}^{\mathscr{C}} / K$. Let then $\bar{S}_{K}$ be the closed Riemann surface obtained from $S_{K}$ filling in its punctures and, for a commutative unitary ring of coefficients $A$, let $H_{1}\left(\bar{S}_{K}, A\right)$ be its first homology group. There is then a natural map $\psi_{K}: K \rightarrow H_{1}\left(\bar{S}_{K}, A\right)$.

For a given $\gamma \in \hat{\mathscr{L}}^{\mathscr{C}}$, let us denote by the same letter an element of the profinite group $\widehat{\Pi}^{\mathscr{C}}$ in the class of the given profinite simple closed curve and let $\nu_{K}(\gamma)$ be the smallest positive integer such that $\gamma^{\nu_{K}(\gamma)} \in K$. For a profinite multicurve $\sigma \in L\left(\widehat{\Pi}^{\mathscr{C}}\right)$, let us also denote by $\sigma$ a subset of $\widehat{\Pi}^{\mathscr{C}}$ in the class of the given multicurve. We let then $V_{K, \sigma}$ be the primitive $A\left[G_{K}\right]$-submodule of $H_{1}\left(\bar{S}_{K}, A\right)$ generated by the $G_{K}$-orbit of $\left\{\psi_{K}\left(\gamma^{\nu_{K}(\gamma)}\right)\right\}_{\gamma \in \sigma}$.

Let $\operatorname{Gr}_{G_{K}}\left(H_{1}\left(\bar{S}_{K}, A\right)\right)$ be the absolute Grassmanian of primitive $A\left[G_{K}\right]$-submodules of the homology group $H_{1}\left(\bar{S}_{K}, A\right)$, that is to say the disjoint union of the Grassmanians of primitive, $k$-dimensional, $A\left[G_{K}\right]$-submodules of $H_{1}\left(\bar{S}_{K}, A\right)$, for all $1 \leq k \leq \operatorname{rank} H_{1}\left(\bar{S}_{K}, A\right)$.

For $A_{p}$ equal to the ring of $p$-adic integers $\mathbb{Z}_{p}$ or the finite field $\mathbb{F}_{p}$, the absolute Grassmanian $\operatorname{Gr}_{G_{K}}\left(H_{1}\left(\bar{S}_{K}, A_{p}\right)\right)$ has a natural structure of profinite space, while, for $A_{p}=\mathbb{Q}_{p}$, it is a locally compact totally disconnected Hausdorff space. In all cases, for $\sigma \in L\left(\widehat{\Pi}^{\mathscr{C}}\right)$, the assignment $\sigma \mapsto V_{K, \sigma}$ defines a natural continuous $\check{\Gamma}(S)^{\mathscr{C}}$-equivariant map:

$$
\Psi_{K, p}: L\left(\widehat{\Pi}^{\mathscr{C}}\right) \rightarrow \operatorname{Gr}_{G_{K}}\left(H_{1}\left(\bar{S}_{K}, A_{p}\right)\right) .
$$

The following theorem is then a straightforward generalization of Theorem 5.6 in [8],

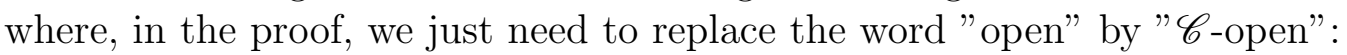

Theorem 4.1. For $p>0$ a prime number, let $A_{p}=\mathbb{F}_{p}, \mathbb{Z}_{p}$ or $\mathbb{Q}_{p}$. There is a natural continuous $\check{\Gamma}(S)^{\mathscr{C}}$-equivariant injective map:

$$
\widehat{\Psi}_{p}:=\prod_{K ্_{\mathscr{C}} \mathscr{C}} \Psi_{K, p}: L\left(\widehat{\Pi}^{\mathscr{C}}\right) \hookrightarrow \prod_{K \unlhd \mathscr{C} \widehat{\Pi}^{\mathscr{C}}} \operatorname{Gr}_{G_{K}}\left(H_{1}\left(\bar{S}_{K}, A_{p}\right)\right) .
$$

\subsection{Centralizers of pro- $\mathscr{C}$ multitwists}

The set $\mathscr{L}$ of isotopy classes of nonperipheral simple closed curves on $S$ parametrizes the set of Dehn twists of $\Gamma(S)$, which is the standard set of generators for this group. The assignment $\gamma \mapsto \tau_{\gamma}$, for $\gamma \in \mathscr{L}$, defines an embedding $d: \mathscr{L} \hookrightarrow \Gamma(S)$, thus relating, in an immediate way, the elementary topology of $S$ with the group structure of $\Gamma(S)$.

The set $\left\{\tau_{\gamma}\right\}_{\gamma \in \mathscr{L}}$ of all Dehn twists of $\Gamma(S)$ is closed under conjugation and falls in a finite set of conjugacy classes which are in bijective correspondence with the possible topological types of the Riemann surface $S \backslash \gamma$. The set of pro- $\mathscr{C}$ Dehn twists is defined to be the closure of the image of the set $\left\{\tau_{\gamma}\right\}_{\gamma \in \mathscr{L}}$ inside $\check{\Gamma}(S)^{\mathscr{C}}$. This is the same as the union of the conjugacy classes in $\check{\Gamma}(S)^{\mathscr{C}}$ of the images of the Dehn twists of $\Gamma(S)$. 
The composition of the embedding $d$ with the natural monomorphism $\Gamma(S) \hookrightarrow \check{\Gamma}(S)^{\mathscr{C}}$ is a $\Gamma(S)$-equivariant map $\mathscr{L} \hookrightarrow \check{\Gamma}(S)^{\mathscr{C}}$, where we let $\Gamma(S)$ act by conjugation on $\check{\Gamma}(S)^{\mathscr{C}}$. From the universal property of the $\breve{\Gamma}(S)^{\mathscr{C}}$-completion and Theorem 3.5, it then follows that this map extends to a continuous $\check{\Gamma}(S)^{\mathscr{C}}$-equivariant map $\hat{d}^{\mathscr{C}}: \hat{\mathscr{L}}^{\mathscr{C}} \rightarrow \check{\Gamma}(S)^{\mathscr{C}}$, whose image is the set of pro- $\mathscr{C}$ Dehn twists. We then let $\tau_{\gamma}:=\hat{d}^{\mathscr{C}}(\gamma)$, for all $\gamma \in \mathscr{L}^{\mathscr{C}}$. Similarly, to a multicurve $\sigma=\left\{\gamma_{1}, \ldots, \gamma_{s}\right\} \in \mathscr{L}^{\mathscr{C}}$ and a multi-index $\left(h_{1}, \ldots, h_{s}\right) \in\left(\hat{\mathbb{Z}}^{\mathscr{C}}\right)^{s}$, we associate the pro- $\mathscr{C}$ multitwist $\tau_{\gamma_{1}}^{h_{1}} \cdot \ldots \cdot \tau_{\gamma_{s}}^{h_{s}}$.

In this section, we want to extend to pro- $\mathscr{C}$ congruence mapping class groups Theorem 6.1 of [8] and then its Corollary 6.2 , which describes the centralizer of a profinite multitwist. We will actually prove a stronger version of those results:

Theorem 4.2. Let $\sigma=\left\{\gamma_{1}, \ldots, \gamma_{s}\right\}$ and $\sigma^{\prime}=\left\{\delta_{1}, \ldots, \delta_{t}\right\}$ be two pro- $\mathscr{C}$ multicurves on the surface $S$. Suppose that, for multi-indices $\left(h_{1}, \ldots, h_{s}\right) \in m_{\sigma} \cdot(\mathbb{Z} \backslash\{0\})^{s}$ and $\left(k_{1}, \ldots, k_{t}\right) \in$ $m_{\sigma^{\prime}} \cdot(\mathbb{Z} \backslash\{0\})^{t}$, where $m_{\sigma}, m_{\sigma^{\prime}} \in\left(\hat{\mathbb{Z}}^{\mathscr{C}}\right)^{*}$, there is an identity:

$$
\tau_{\gamma_{1}}^{h_{1}} \cdot \ldots \cdot \tau_{\gamma_{s}}^{h_{s}}=\tau_{\delta_{1}}^{k_{1}} \cdot \ldots \cdot \tau_{\delta_{t}}^{k_{t}} \in \check{\Gamma}(S)^{\mathscr{C}}
$$

Then, we have:

(i) $t=s$;

(ii) there is a permutation $\phi \in \Sigma_{s}$ such that $\delta_{i}=\gamma_{\phi(i)}$ and $k_{i}=h_{\phi(i)}$, for $i=1, \ldots, s$.

Proof. By Theorem 4.1, it is enough to show that, for a proper characteristic $\mathscr{C}$-open subgroup $K$ of $\widehat{\Pi}^{\mathscr{C}}$, a pro- $\mathscr{C}$ multitwist $\tau_{\gamma_{1}}^{h_{1}} \cdot \ldots \cdot \tau_{\gamma_{s}}^{h_{s}}$ determines the $\mathbb{Q}_{p}$-subspace $V_{K, \sigma}$ of $H_{1}\left(\bar{S}_{K}, \mathbb{Q}_{p}\right)$, where $\sigma=\left\{\gamma_{1}, \ldots, \gamma_{s}\right\}$.

Let $N_{\check{\Gamma}\left(S_{K}\right)^{\mathscr{C}}}\left(G_{K}\right)$ be the normalizer of the finite group $G_{K}$ in the pro- $\mathscr{C}$ congruence mapping class group $\check{\Gamma}\left(S_{K}\right)$. There is an exact sequence (cf. Section 2 in [5]. Note that here we are considering pure mapping class groups):

$$
1 \rightarrow G_{K} \rightarrow N_{\check{\Gamma}\left(S_{K}\right)^{\mathscr{C}}}\left(G_{K}\right) \rightarrow \check{\Gamma}(S)^{\mathscr{C}},
$$

where the image of $N_{\check{\Gamma}\left(S_{K}\right)^{\mathscr{C}}}\left(G_{K}\right)$ in $\check{\Gamma}(S)^{\mathscr{C}}$ is an open normal subgroup.

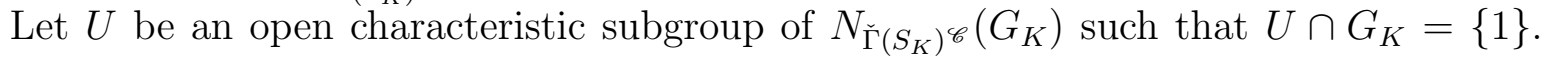
Then, $U$ identifies with an open normal subgroup of $\check{\Gamma}(S)^{\mathscr{C}}$. Since the $U$-orbit of $\sigma$ is open inside its $\check{\Gamma}(S)^{\mathscr{C}}$-orbit, the $U$-orbit of $\sigma$ contains an element in the image of $C(S)$. There is then an $f \in U$ such that $f(\sigma) \in C(S)$ and, for such element, there holds $V_{K, f(\sigma)}=\bar{f}\left(V_{K, \sigma}\right)$, where $\bar{f}$ is the image of $f$ in the symplectic group $\operatorname{Sp}\left(H_{1}\left(\bar{S}_{K}, \mathbb{Q}_{p}\right)\right)$. Therefore, in order to prove the above claim, it is enough to consider the case when $\sigma \in C(S) \subset L\left(\widehat{\Pi}^{\mathscr{C}}\right)$.

There is an $r \in \mathbb{N}^{+}$such that, for every simple closed curve $\gamma$ on $S$, we have that $\tau_{\gamma}^{r} \in U \cap \Gamma(S)$. From our hypothesis on the multi-index $\left(h_{1}, \ldots, h_{s}\right)$, it follows that there is an $m \in \hat{\mathbb{Z}}^{\mathscr{C}}$ such that $m h_{i}$ is a positive integer divisible by $r$, for all $i=1, \ldots, s$, and then such that $\tau_{\gamma_{1}}^{m h_{1}} \cdot \ldots \cdot \tau_{\gamma_{s}}^{m h_{s}} \in U \cap \Gamma(S)$.

Since $U \cap G_{K}=\{1\}$, the subgroup $U$ is contained in the centralizer $Z_{\check{\Gamma}\left(S_{K}\right)^{\mathscr{E}}}\left(G_{K}\right)$ of $G_{K}$ in $\check{\Gamma}\left(S_{K}\right)$ and so there is a natural representation $\rho_{U}: U \cap \Gamma(S) \rightarrow Z_{\operatorname{Sp}\left(H_{1}\left(\bar{S}_{K}, \mathbb{Q}\right)\right)}\left(G_{K}\right)$. 
In order to prove our claim and the theorem, it is then enough to show that we can recover the subspace $V_{K, \sigma}^{\mathbb{Q}}:=V_{K, \sigma} \cap H_{1}\left(\bar{S}_{K}, \mathbb{Q}\right)$ of $H_{1}\left(\bar{S}_{K}, \mathbb{Q}\right)$ from the multitransvection $\rho_{U}\left(\tau_{\gamma_{1}}^{m h_{1}} \cdot \ldots \cdot \tau_{\gamma_{s}}^{m h_{s}}\right)$. The logarithm of this multitransvection is the symmetric bilinear form on $H_{1}\left(\bar{S}_{K}, \mathbb{Q}\right)$ (cf. Section 1 in [17]) defined by:

$$
\log \left(\rho_{U}\left(\tau_{\gamma_{1}}^{m h_{1}} \cdot \ldots \cdot \tau_{\gamma_{s}}^{m h_{s}}\right)\right)=\sum_{i=1}^{s} \nu_{i} \sum_{\tilde{\gamma} / \gamma_{i}}\left\langle[\tilde{\gamma}],{ }_{-}\right\rangle_{\bar{S}_{K}} \in \operatorname{Sym}^{2}\left(H^{1}\left(\bar{S}_{K}, \mathbb{Q}\right)\right)
$$

where the second sum runs over the simple closed curves $\tilde{\gamma}$ on $S_{K}$ which cover $\gamma_{i}$, we let $\nu_{i}:=m h_{i} / \operatorname{deg}\left(\tilde{\gamma} / \gamma_{i}\right)$, for $i=1, \ldots, s$, and we denote by $[\tilde{\gamma}]$ a cycle in $H_{1}\left(\bar{S}_{K}, \mathbb{Q}\right)$ supported on $\tilde{\gamma}$ and by $\left\langle_{-},\right\rangle_{\bar{S}_{K}}$ the standard symplectic bilinear form on $H_{1}\left(\bar{S}_{K}, \mathbb{Q}\right)$.

In Lemma 5.11 of [6], we showed that, when $\log \left(\rho_{U}\left(\tau_{\gamma_{1}}^{m h_{1}} \cdot \ldots \cdot \tau_{\gamma_{s}}^{m h_{s}}\right)\right)$ is a semidefinite symmetric bilinear form (e.g. $\nu_{i} \in \mathbb{N}^{+}$, for all $i=1, \ldots, s$ ), it is possible to recover the subspace $V_{K, \sigma}^{\mathbb{Q}}$ as the core (cf. ibidem) of this form.

However, it is not difficult to show that this is true also without that assumption. Indeed, for $K$ a proper characteristic subgroup of $\widehat{\Pi}^{\mathscr{C}}$, the set $\{\tilde{\gamma}\}$ of simple closed curves lying over a fixed $\gamma_{i}$ disconnects the surface $S_{K}$, for every $i=1, \ldots, s$. There are then subspaces $W^{+}$and $W^{-}$of $H_{1}\left(\bar{S}_{K}, \mathbb{Q}\right)$, nondegenerate for the symplectic form $\left\langle{ }_{-},\right\rangle_{\bar{S}_{K}}$, with the property that $V_{K, \sigma}^{\mathbb{Q}}=\left(W^{+} \cap V_{K, \sigma}^{\mathbb{Q}}\right)+\left(W^{-} \cap V_{K, \sigma}^{\mathbb{Q}}\right)$ and such that the restriction of $\log \left(\rho_{U}\left(\tau_{\gamma_{1}}^{m h_{1}} \cdot \ldots \cdot \tau_{\gamma_{s}}^{m h_{s}}\right)\right)$ to $W^{+}$and $W^{-}$is semidefinite. The same argument of Lemma 5.11 of [6] then applies to show that we can recover the subspaces $V_{K, \sigma} \cap W^{ \pm}$from such restriction and hence the subspace $V_{K, \sigma}^{\mathbb{Q}}$ as their sum.

From the identity $f \cdot\left(\tau_{\gamma_{1}}^{h_{1}} \cdot \ldots \cdot \tau_{\gamma_{k}}^{h_{k}}\right) \cdot f^{-1}=\tau_{f\left(\gamma_{1}\right)}^{h_{1}} \cdot \ldots \cdot \tau_{f\left(\gamma_{k}\right)}^{h_{k}}$, it then follows at once:

Corollary 4.3. Let $\sigma=\left\{\gamma_{1}, \ldots, \gamma_{s}\right\}$ be a pro-C $\mathscr{C}$ multicurve on $S$ and $\left(h_{1}, \ldots, h_{k}\right) \in$ $m_{\sigma} \cdot(\mathbb{Z} \backslash\{0\})^{k}$ a multi-index, where $m_{\sigma} \in\left(\hat{\mathbb{Z}}^{\mathscr{C}}\right)^{*}$. Then, we have

$$
Z_{\check{\Gamma}(S)^{\mathscr{C}}}\left(\tau_{\gamma_{1}}^{h_{1}} \cdot \ldots \cdot \tau_{\gamma_{k}}^{h_{k}}\right)=N_{\check{\Gamma}(S)^{\mathscr{C}}}\left(\left\langle\tau_{\gamma_{1}}^{h_{1}} \cdot \ldots \cdot \tau_{\gamma_{k}}^{h_{k}}\right\rangle\right)=N_{\check{\Gamma}(S)^{\mathscr{C}}}\left(\left\langle\tau_{\gamma_{1}}, \ldots, \tau_{\gamma_{k}}\right\rangle\right)=\check{\Gamma}(S)_{\sigma}^{\mathscr{C}},
$$

where $\check{\Gamma}(S)_{\sigma}^{\mathscr{C}}$ is the stabilizer of $\sigma$ described in Theorem 3.6.

\subsection{The center of the pro- $\mathscr{C}$ congruence mapping class group}

Theorem 4.4. Let $S=S_{g, n}$ be a surface of negative Euler characteristic. For every open subgroup $U$ of $\check{\Gamma}(S)^{\mathscr{C}}$, we then have:

$$
Z_{\check{\Gamma}(S)^{\mathscr{C}}}(U)=Z\left(\check{\Gamma}(S)^{\mathscr{C}}\right)=Z(\Gamma(S)) .
$$

Thus, all these groups are trivial for $(g, n) \neq(1,1),(2,0)$. Otherwise, they are generated by the hyperelliptic involution. 
Proof. Let us prove the identity $Z_{\check{\Gamma}(S)^{\mathscr{C}}}(U)=Z\left(\check{\Gamma}(S)^{\mathscr{C}}\right)$. Since $\check{\Gamma}(S)^{\mathscr{C}}$ is topologically generated by a finite set of Dehn twists $\left\{\tau_{\gamma}\right\}_{\gamma \in I}$ and there is an $h_{U} \in \mathbb{N}^{+}$such that $\tau_{\gamma}^{h_{U}} \in U$, for all $\gamma \in I$, by Corollary 4.3, there is a series of identities and an inclusion:

$$
Z\left(\check{\Gamma}(S)^{\mathscr{C}}\right)=Z\left(\left\langle\tau_{\gamma}\right\rangle_{\gamma \in I}\right)=Z\left(\left\langle\tau_{\gamma}^{h_{U}}\right\rangle_{\gamma \in I}\right) \supseteq Z_{\check{\Gamma}(S)^{\mathscr{C}}}(U)
$$

The other inclusion $Z\left(\check{\Gamma}(S)^{\mathscr{C}}\right) \subseteq Z_{\check{\Gamma}(S)^{\mathscr{C}}}(U)$ is trivial, hence the conclusion follows.

In order to prove the identity $Z\left(\check{\Gamma}(S)^{\mathscr{C}}\right)=Z(\Gamma(S))$, we need to consider first the case $g=0$. We treat this case by induction on $n \geq 3$. The case $n=3$ is trivial because, for $n=3$, the group $\Gamma(S)$ is trivial. Let us then assume that the theorem holds for $3 \leq n \leq k$ and let us prove it for $n=k+1$. Let $\gamma$ be a simple closed curve on $S$ which separates two of the punctures on $S$ from the others. Then, we have $Z\left(\check{\Gamma}(S)^{\mathscr{C}}\right) \subseteq Z_{\check{\Gamma}(S)^{\mathscr{C}}}\left(\tau_{\gamma}\right)$. Since $Z(\Gamma(S)) \cap\left\langle\tau_{\gamma}\right\rangle=\{1\}$ and $\check{\Gamma}(S)_{\gamma}^{\mathscr{C}}=\check{\Gamma}(S)_{\vec{\gamma}}^{\mathscr{C}}$ (because we are considering pure mapping class groups), by Corollary 4.3 , the center $Z\left(\check{\Gamma}(S)^{\mathscr{C}}\right)$ embeds in the center of $\check{\Gamma}(S \backslash \gamma)^{\mathscr{C}}$. Now, the induction hypothesis implies that the latter is trivial and so the conclusion follows.

For $g=1, n=1$, we have to prove that $Z\left(\check{\Gamma}(S)^{\mathscr{C}}\right)=Z(\Gamma(S))=\langle\iota\rangle$, where $\iota$ is the elliptic involution. Let $\gamma$ be a nonseparating closed curve on $S$. Then, by Corollary 4.3, $Z\left(\check{\Gamma}(S)^{\mathscr{C}}\right) \subseteq Z\left(\check{\Gamma}_{\gamma}^{\mathscr{C}}\right)$. Given $f \in Z\left(\check{\Gamma}(S)^{\mathscr{C}}\right)$, possibly after replacing $f$ with $f \cdot \iota$, we can assume that actually $f \in \check{\Gamma}_{\vec{\gamma}}^{\mathscr{C}}$. But then, since $Z(\Gamma(S)) \cap\left\langle\tau_{\gamma}\right\rangle=\{1\}$, the element $f$ can be identified with an element of the center of $\check{\Gamma}(S \backslash \gamma)^{\mathscr{C}}$, which, by the genus 0 case, is trivial. Therefore, $f$ is either trivial or equal to $\iota$, which proves the claim.

For $g=1, n \geq 2$, we have to prove that $Z\left(\check{\Gamma}(S)^{\mathscr{C}}\right)=Z(\Gamma(S))=\{1\}$. Let again $\gamma$ be a nonseparating closed curve on $S$. Then, by Corollary $4.3, Z\left(\check{\Gamma}(S)^{\mathscr{C}}\right) \subseteq Z\left(\check{\Gamma}_{\gamma}^{\mathscr{C}}\right)=Z\left(\check{\Gamma}_{\vec{\gamma}}^{\mathscr{C}}\right)$. As above, by the genus 0 case, this implies that the center of $\check{\Gamma}(S)^{\mathscr{C}}$ is trivial.

For $g=2, n=0$, we have to prove that $Z\left(\check{\Gamma}(S)^{\mathscr{C}}\right)=Z(\Gamma(S))=\langle\iota\rangle$, where $\iota$ is the hyperelliptic involution. Let $\gamma$ be a nonseparating closed curve on $S$ and $f \in Z\left(\check{\Gamma}(S)^{\mathscr{C}}\right)$. As above, possibly after replacing $f$ with $f \cdot \iota$, we have that $f \in \check{\Gamma}_{\vec{\gamma}}^{\mathscr{C}}$. We then proceed as in the case $g=1, n=1$ and show that either $f$ is trivial or $f=\iota$.

For $g=2, n \geq 1$, we have to prove that $Z\left(\check{\Gamma}(S)^{\mathscr{C}}\right)=Z(\Gamma(S))=\{1\}$. In this case, we let $\gamma$ be a separating curve on $S$ which bounds an unpunctured genus 1 subsurface $S^{\prime}$ of $S$. By Corollary 4.3, we have $Z\left(\check{\Gamma}(S)^{\mathscr{C}}\right) \subseteq Z\left(\check{\Gamma}_{\gamma}^{\mathscr{C}}\right)=Z\left(\check{\Gamma}_{\vec{\gamma}}^{\mathscr{C}}\right)$. Let us show that $Z\left(\check{\Gamma}_{\vec{\gamma}}^{\mathscr{C}}\right)$ consists of the $\hat{\mathbb{Z}}^{\mathscr{C}}$-powers of the half-twist $\tau_{1 / 2}$ on $S$ which restricts to the elliptic involution on $S^{\prime}$ and to the identity on the complement of $S^{\prime}$ in $S$. By the genus 1 case, indeed, the center of $\check{\Gamma}(S \backslash \gamma)^{\mathscr{C}}$ is generated by the homeomorphism $\iota$ which restricts to the elliptic involution on $S^{\prime}$ and to the identity on the complement of $S^{\prime}$ in $S$. This implies the previous claim since $\tau_{1 / 2}^{2}=\tau_{\gamma}$. The conclusion then follows because $Z(\Gamma(S)) \cap\left\langle\tau_{1 / 2}\right\rangle=\{1\}$.

For $g \geq 3$, we proceed by induction on the genus taking as basis for the induction the cases already proved. Let us then assume that the statement of the theorem holds for all $2 \leq g \leq k$ and let us prove it for $g=k+1$. Essentially the same argument of the case $g=2, n \geq 1$ and the induction hypothesis yield that $Z\left(\check{\Gamma}(S)^{\mathscr{C}}\right)=Z(\Gamma(S))=\{1\}$. 


\subsection{A Birman exact sequence for pro- $\mathscr{C}$-congruence mapping class groups: the case $\check{\Pi}^{\mathscr{C}} \equiv \widehat{\Pi}^{\mathscr{C}}$}

Let us now describe in a more precise way the epimorphism $\hat{\Delta}_{x}^{\mathscr{C}}: \check{\Gamma}\left(S_{x}\right)^{\mathscr{C}} \rightarrow \check{\Gamma}(S, x)^{\mathscr{C}}$ introduced in Section 2.2 and give necessary and sufficient conditions for this to be an isomorphism. An immediate consequence of Corollary 4.3 and Theorem 4.4 is:

Corollary 4.5. Let $S$ be a connected surface of negative Euler characteristic, $x \in S$ and let $\check{\Pi}^{\mathscr{C}}$ be the $\mathscr{C}$-congruence completion of $\Pi:=\pi_{1}(S, x)$ defined in Section 2.1. The action by restriction of inner automorphisms of $\check{\Gamma}\left(S_{x}\right)^{\mathscr{C}}$ on its normal subgroup $\check{\Pi}^{\mathscr{C}}$ then induces a faithful representation $\tilde{\rho}_{\mathscr{C}}: \check{\Gamma}\left(S_{x}\right)^{\mathscr{C}} \hookrightarrow \operatorname{Aut}\left(\check{\Pi}^{\mathscr{C}}\right)$. In particular, the homomorphism $\hat{\Delta}_{x}^{\mathscr{C}}$ is an isomorphism, if and only if, $\check{\Pi}^{\mathscr{C}} \equiv \widehat{\Pi}^{\mathscr{C}}$.

Proof. Let $\gamma \in \Pi \subset \check{\Pi}^{\mathscr{C}}$ be an element whose free homotopy class contains a simple closed curve on $S$, which we also denote by $\gamma$. Then, its image in $\Gamma\left(S_{x}\right) \subset \check{\Gamma}\left(S_{x}\right)^{\mathscr{C}}$ is the bounding pair map $\tau_{\gamma^{+}} \cdot \tau_{\gamma^{-}}^{-1}$, where $\gamma^{+}, \gamma^{-}$is a pair of simple closed curves on $S_{x}$ which bound a tubular neighborhood of $\gamma$. Let $f \in \operatorname{ker} \tilde{\rho}_{\mathscr{C}}$. By the definition of $\tilde{\rho}_{\mathscr{C}}$, the element $f$ centralizes the multitwist $\tau_{\gamma^{+}} \cdot \tau_{\gamma^{-}}^{-1}$. By Corollary 4.3, it then centralizes both Dehn twists $\tau_{\gamma^{+}}, \tau_{\gamma^{-}}^{-1}$. Since, for all $\gamma \in \Pi$ as above, these elements generate (topologically) $\check{\Gamma}\left(S_{x}\right)^{\mathscr{C}}$, it follows that $f \in Z\left(\check{\Gamma}\left(S_{x}\right)^{\mathscr{C}}\right)$. Thus, either $f=1$ or $f$ is an elliptic or hyperelliptic involution. But, in the latter case, $\tilde{\rho}_{\mathscr{C}}(f) \neq 1$. Therefore, $f=1$. The last claim of the corollary is obvious.

Remark 4.6. Since a group acts faithfully on itself by inner automorphisms if and only if it is centerfree, Corollary 4.5 also implies that the profinite group $\check{\Pi}^{\mathscr{C}}$ is centerfree.

Corollary 4.7. Let $S$ be an oriented connected surface of finite negative Euler characteristic, $x \in S$ and $\mathscr{C}$ a class of finite groups such that $\check{\Pi}^{\mathscr{C}} \equiv \widehat{\Pi}^{\mathscr{C}}$ (e.g. $\mathscr{C}=\mathscr{F}$ or $(p)$, for $p$ a prime). Then, $\hat{\Delta}_{x}^{\mathscr{C}}: \check{\Gamma}\left(S_{x}\right)^{\mathscr{C}} \rightarrow \check{\Gamma}(S, x)^{\mathscr{C}}$ is an isomorphism and determines a Birman exact sequence:

$$
1 \rightarrow \widehat{\Pi}^{\mathscr{C}} \rightarrow \check{\Gamma}\left(S_{x}\right)^{\mathscr{C}} \rightarrow \check{\Gamma}(S)^{\mathscr{C}} \rightarrow 1
$$

Proof. By Corollary 4.5, the homomorphism $\hat{\Delta}_{x}^{\mathscr{C}}$ is an isomorphism. Combining this isomorphism with the Birman exact sequence (1), we get the short exact sequence (3). The claim for $\mathscr{C}=\mathscr{F}$ or $(p)$ is then just (ii) of Proposition 2.4.

Remark 4.8. For $\mathscr{C}=\mathscr{F}$ or $(p)$, Corollary 4.7 was proved by Matsumoto in the open surface case (cf. Theorem 2.2 in [18]) and then extended by Hoshi and Mochizuki to the closed surface case (cf. Corollary 6.2 in [15] and Lemma 20 in [13]).

\subsection{Comparison of $\mathscr{C}$-congruence topologies}

In Corollary 4.5, we gave necessary and sufficient conditions under which the $\mathscr{C}$-congruence topologies introduced in Section 2.1 coincide. In this section we prove a similar result for 
the $\mathscr{C}$-congruence topologies of Section 2.3. The conclusion will be that all the possible $\mathscr{C}$-congruence topologies defined on the mapping class group coincide for $\mathscr{C}=\mathscr{F}$ or $(p)$.

With the notations of Section 2.3, let us denote by $\pi_{i}: S(n) \rightarrow S(n-1)$ the natural map which forgets the $i$-th point, for $i=1, \ldots, n$. The fiber of $\pi_{i}$ over the point $\bar{x}_{i}:=$ $\left\{x_{1}, \ldots, x_{i-1}, x_{i+1}, \ldots, x_{n}\right\} \in S(n-1)$ is then naturally identified with $S_{\bar{x}_{i}}:=S \backslash \bar{x}_{i}$ and we let $\Pi_{\bar{x}_{i}}:=\pi_{1}\left(S_{\bar{x}_{i}}, x_{i}\right)$, for $i=1, \ldots, n$. There is a natural $\Gamma(S, \bar{x})$-equivariant embedding $\Pi_{\bar{x}_{i}} \hookrightarrow \Pi(n)$ and also $\Pi_{\bar{x}_{i}} \hookrightarrow \Gamma\left(S_{\bar{x}}\right)$, for $i=1, \ldots, n$. We then denote by $\check{\Pi}_{\bar{x}_{i}}^{\mathscr{C}}$ the closure of $\Pi_{\bar{x}_{i}}$ in the $\mathscr{C}$-congruence completion $\check{\Gamma}\left(S_{\bar{x}}\right)^{\mathscr{C}}$. The following result, for $\mathscr{C}=\mathscr{F}$ or $(p)$, was proved by Mochizuki and Tamagawa (cf. (i) of Proposition 2.2 [19]):

Proposition 4.9. Let $S$ be a connected oriented surface of finite type, $S(n)$ the configuration space of $n$ distinct point on it, let $\bar{x}=\left\{x_{1}, \ldots, x_{n}\right\} \in S(n)$ and let $\mathscr{C}$ be a class of finite groups such that $\check{\Pi}_{\bar{x}_{i}}^{\mathscr{C}} \equiv \widehat{\Pi}_{\bar{x}_{i}}^{\mathscr{C}}$, for all $n \geq 1$ (e.g. $\mathscr{C}=\mathscr{F}$ or $(p)$ ). Then, any projection morphism $\pi_{i}: S(n) \rightarrow S(n-1)$ determines a natural short exact sequence of pro- $\mathscr{C}$ fundamental groups, for $i=1, \ldots, n$ :

$$
1 \rightarrow \widehat{\Pi}_{\bar{x}_{i}}^{\mathscr{C}} \rightarrow \hat{\pi}_{1}(S(n), \bar{x})^{\mathscr{C}} \rightarrow \hat{\pi}_{1}\left(S(n-1), \bar{x}_{i}\right)^{\mathscr{C}} \rightarrow 1 .
$$

Proof. Let $\Pi(n):=\pi_{1}(S(n), \bar{x})$ and $\Pi(n-1):=\pi_{1}\left(S(n-1), \bar{x}_{i}\right)$. There is a natural embedding $\Pi(n) \subset \Gamma(S, \bar{x}) \cong \Gamma\left(S_{\bar{x}}\right)$ and we identify $\Pi(n)$ with a subgroup of $\Gamma\left(S_{\bar{x}}\right)$. By induction on $n \geq 1$, we will then prove the following statement:

- The closure $\bar{\Pi}(n)$ of $\Pi(n)$ in the $\mathscr{C}$-congruence completion $\check{\Gamma}\left(S_{\bar{x}}\right)^{\mathscr{C}}$ is the pro- $\mathscr{C}$ completion $\widehat{\Pi}(n)^{\mathscr{C}}$.

The case $n=1$ is just that $\widehat{\Pi}(1)^{\mathscr{C}}=\widehat{\Pi}^{\mathscr{C}} \equiv \check{\Pi}^{\mathscr{C}} \subset \check{\Gamma}\left(S_{x_{1}}\right)^{\mathscr{C}}$, which we know by hypothesis. So, let us assume that the closure $\bar{\Pi}(n-1)$ of $\Pi(n-1)$ in $\check{\Gamma}\left(S_{\bar{x}_{i}}\right)^{\mathscr{C}}$ is the pro- $\mathscr{C}$ completion $\widehat{\Pi}(n-1)^{\mathscr{C}}$ and let us prove the same statement for $\Pi(n)$.

The isomorphisms $\Gamma(S, \bar{x}) \cong \Gamma\left(S_{\bar{x}}\right)$ and $\Gamma\left(S, \bar{x}_{i}\right) \cong \Gamma\left(S_{\bar{x}_{i}}\right)$ identify, respectively, the group $\Pi(n)$ with a subgroup of $\Gamma\left(S_{\bar{x}}\right)$, the group $\Pi(n-1)$ with a subgroup of $\Gamma\left(S_{\bar{x}_{i}}\right)$ and the group $\Pi_{\bar{x}_{i}}$ with the kernel of the natural homomorphism $\Gamma\left(S_{\bar{x}}\right) \rightarrow \Gamma\left(S_{\bar{x}_{i}}\right)$.

By hypothesis, the closure of $\Pi_{\bar{x}_{i}}$ in the $\mathscr{C}$-congruence completion $\check{\Gamma}\left(S_{\bar{x}_{i}}\right)^{\mathscr{C}}$ is the pro- $\mathscr{C}$ completion $\widehat{\Pi}_{\bar{x}_{i}}^{\mathscr{C}}$. Therefore, by the inductive hypothesis and Corollary 4.7 , there is a short exact sequence:

$$
1 \rightarrow \widehat{\Pi}_{\bar{x}_{i}}^{\mathscr{C}} \rightarrow \bar{\Pi}(n) \rightarrow \widehat{\Pi}(n-1)^{\mathscr{C}} \rightarrow 1 .
$$

This immediately implies the claim that $\bar{\Pi}(n)=\widehat{\Pi}(n)^{\mathscr{C}}$. The statement of the proposition then follows from the short exact sequence (5).

Corollary 4.10. Let $\mathscr{C}$ be a class of finite groups such that $\check{\Pi}_{\bar{x}_{i}}^{\mathscr{C}} \equiv \widehat{\Pi}_{\bar{x}_{i}}^{\mathscr{C}}$, for all $n \geq 1$ (e.g. $\mathscr{C}=\mathscr{F}$ or $(p)$ ). Then, there are natural isomorphisms of profinite groups $\check{\Gamma}(S, \bar{x})^{\mathscr{C}} \cong$ $\check{\Gamma}\left(S_{\bar{x}_{i}}, x_{i}\right)^{\mathscr{C}} \cong \check{\Gamma}\left(S_{\bar{x}}\right)^{\mathscr{C}}$, where $\bar{x}=\left\{x_{1}, \ldots, x_{n}\right\} \in S(n)$ and $i=1, \ldots, n$.

Proof. In the proof of Proposition 4.9, we have shown that the closure of $\Pi(n)$ in $\check{\Gamma}\left(S_{\bar{x}}\right)^{\mathscr{C}}$ is the pro- $\mathscr{C}$ completion $\widehat{\Pi}(n)^{\mathscr{C}}$. Let us fix $i \in\{1, \ldots, n\}$. By Corollary 4.7, there is a 
natural isomorphism $\check{\Gamma}\left(S_{\bar{x}}\right)^{\mathscr{C}} \cong \check{\Gamma}\left(S_{\bar{x}_{i}}, x_{i}\right)^{\mathscr{C}}$. Therefore, the closure of $\Pi(n)$ in $\check{\Gamma}\left(S_{\bar{x}_{i}}, x_{i}\right)^{\mathscr{C}}$ also identifies with the pro- $\mathscr{C}$ completion $\widehat{\Pi}(n)^{\mathscr{C}}$. There is then a natural continuous epimorphism $\phi: \check{\Gamma}\left(S_{\bar{x}_{i}}, x_{i}\right)^{\mathscr{C}} \rightarrow \check{\Gamma}(S, \bar{x})^{\mathscr{C}}$, obtained restricting the inner automorphisms of $\check{\Gamma}\left(S_{\bar{x}_{i}}, x_{i}\right)^{\mathscr{C}}$ to its normal subgroup $\widehat{\Pi}(n)^{\mathscr{C}}$. On the other hand, by Proposition 4.9 , the closure of $\Pi_{\bar{x}_{i}}$ in the $\mathscr{C}$-congruence completion $\check{\Gamma}(S, \bar{x})^{\mathscr{C}}$ is the pro- $\mathscr{C}$ completion $\widehat{\Pi}_{\bar{x}_{i}}^{\mathscr{C}}$. Thus, by restricting the inner automorphisms of $\check{\Gamma}(S, \bar{x})^{\mathscr{C}}$ to its normal subgroup $\widehat{\Pi}_{\bar{x}}^{\mathscr{C}}$, we see that there is also a natural continuous epimorphism $\psi: \check{\Gamma}(S, \bar{x})^{\mathscr{C}} \rightarrow \check{\Gamma}\left(S_{\bar{x}_{i}}, x_{i}\right)^{\mathscr{C}}$. It is clear that $\psi \circ \phi$ and $\phi \circ \psi$ are the identity when restricted to the dense subgroups $\Gamma\left(S_{\bar{x}_{i}}, x_{i}\right)$ and $\Gamma(S, \bar{x})$, respectively. Therefore, by continuity, we have $\psi \circ \phi=\operatorname{Id}$ and $\phi \circ \psi=\mathrm{Id}$, which proves the corollary.

Remark 4.11. From the proof of Corollary 4.10, it follows that if the short sequence (4) is exact, then $\check{\Pi}_{\bar{x}_{i}}^{\mathscr{C}} \equiv \widehat{\Pi}_{\bar{x}_{i}}^{\mathscr{C}}$. Since the sequence (4) is right exact for any class of finite groups $\mathscr{C}$, we see that the obstruction to have an isomorphism $\check{\Pi}_{\bar{x}_{i}}^{\mathscr{C}} \equiv \widehat{\Pi}_{\bar{x}_{i}}^{\mathscr{C}}$, for an arbitrary class $\mathscr{C}$ of finite groups, is the fact that the sequence (4) may not be left exact. Necessary and sufficient conditions which ensure left exactness of the pro- $\mathscr{C}$ completion functor are given by Anderson in [1] and are not difficult to check for $\mathscr{C}=\mathscr{F}$ or $(p)$ but are not necessarily satisfied by a more general class of finite groups (e.g. by $\mathscr{C}=\mathscr{S})$.

\section{A Birman exact sequence for pro- $\mathscr{C}$-congruence mapping class groups: the general case}

In this Section, we will show that, for an open surface $S$, the kernel of the epimorphism $\hat{\Delta}_{x}^{\mathscr{C}}: \check{\Gamma}\left(S_{x}\right)^{\mathscr{C}} \rightarrow \check{\Gamma}(S, x)^{\mathscr{C}}$ equals the kernel of the restriction of this map to $\check{\Pi}^{\mathscr{C}}$.

Definition 5.1. With the notations of Section 2.2, let $\Gamma\left(S_{\circ}, \partial S_{\circ}\right)$ be the mapping class group of self-homeomorphisms of $S_{\circ}$ which fix pointwise the boundary $\partial S_{\circ}$. The $\mathscr{C}$ congruence completion $\check{\Gamma}\left(S_{\circ}, \partial S_{\circ}\right)^{\mathscr{C}}$ of $\Gamma\left(S_{\circ}, \partial S_{\circ}\right)$ is the profinite completion induced by the $\mathscr{C}$-topology on $\Pi_{\circ}$ via the faithful representation: $\rho_{\circ}: \Gamma\left(S_{\circ}, \partial S_{\circ}\right) \hookrightarrow \operatorname{Aut}\left(\Pi_{\circ}\right)$.

There is a natural (in general non-split) short exact sequence:

$$
1 \rightarrow \tau_{u}^{\mathbb{Z}} \rightarrow \Gamma\left(S_{\circ}, \partial S_{\circ}\right) \rightarrow \Gamma\left(S_{\circ}\right) \rightarrow 1,
$$

where $\tau_{u}$ is the Dehn twist around the boundary component $\partial S_{\circ}$, and a natural representation:

$$
\check{\rho}^{\mathscr{C}}: \check{\Gamma}\left(S_{\circ}, \partial S_{\circ}\right)^{\mathscr{C}} \rightarrow \operatorname{Out} \mathscr{U}\left(\widehat{\Pi}_{\circ}^{\mathscr{C}}\right) .
$$

By composing the representation $\check{\rho}^{\mathscr{C}}$ with the isomorphism $\hat{\mathrm{K}}_{u}^{\mathscr{C}}$, defined in the proof of Lemma 2.2, we see that the kernel of $\check{\rho}^{\mathscr{C}}$ is precisely the procyclic subgroup generated by the Dehn twist $\tau_{u}$, whose image in $\operatorname{Aut}\left(\widehat{\Pi}_{\circ}^{\mathscr{C}}\right)$ is $\operatorname{inn}(u)$. Therefore, there is also a natural short exact sequence:

$$
1 \rightarrow \tau_{u}^{\hat{\mathbb{Z}}^{\mathscr{C}}} \rightarrow \check{\Gamma}\left(S_{\circ}, \partial S_{\circ}\right)^{\mathscr{C}} \rightarrow \check{\Gamma}\left(S_{\circ}\right)^{\mathscr{C}} \rightarrow 1
$$


Let us now assume that the Riemann surface $S_{\circ}$ is nonclosed and let us consider the map $p: S_{\circ} \hookrightarrow S_{\circ, P}$ obtained filling in a puncture of $S_{\circ}$ with a point $P$. Let $\Pi_{P}:=\pi_{1}\left(S_{\circ, P}, P\right)$. Then, there is an associated Birman short exact sequence:

$$
1 \rightarrow \Pi_{P} \rightarrow \Gamma\left(S_{\circ}, \partial S_{\circ}\right) \stackrel{\Psi}{\rightarrow} \Gamma\left(S_{\circ, P}, \partial S_{\circ, P}\right) \rightarrow 1
$$

The map $p$ has a homotopy section $s: S_{\circ, P} \hookrightarrow S_{\circ}$ such that $\delta:=s\left(\partial S_{\circ, P}\right)$ is a separating simple closed curve on $S_{\circ}$ bounding, on one side, only the boundary $\partial S_{\circ}$ and the puncture labeled by $P$. Let us also fix a path from $x_{\circ}$ to $s(P)$, crossing only once $\delta$, so that the section $s: S_{\circ, P} \hookrightarrow S_{\circ}$ induces a monomorphism on fundamental groups $\Pi_{P} \hookrightarrow \Pi_{\circ}$ and, by Lemma 3.2 , also of their pro- $\mathscr{C}$ completions $\widehat{\Pi}_{P}^{\mathscr{C}} \hookrightarrow \widehat{\Pi}_{\circ}^{\mathscr{C}}$. Let us identify the groups $\Pi_{P}$ and $\widehat{\Pi}_{P}^{\mathscr{C}}$ with their images in $\Pi_{\circ}$ and $\widehat{\Pi}_{\circ}^{\mathscr{C}}$ respectively.

The map $s$ also induces a section $s_{*}: \Gamma\left(S_{\circ, P}, \partial S_{\circ, P}\right) \rightarrow \Gamma\left(S_{\circ}, \partial S_{\circ}\right)$ of the epimorphism $\Psi: \Gamma\left(S_{\circ}, \partial S_{\circ}\right) \rightarrow \Gamma\left(S_{\circ, P}, \partial S_{\circ, P}\right)$ such that the action of the subgroup $s_{*}\left(\Gamma\left(S_{\circ, P}, \partial S_{\circ, P}\right)\right)$ of $\Gamma\left(S_{\circ}, \partial S_{\circ}\right)$ on $\Pi_{\circ}$, via the representation $\rho_{\circ}$, stabilizes the subgroup $\Pi_{P}$. The Birman exact sequence $(7)$ then splits and we have a semidirect product decomposition:

$$
\Gamma\left(S_{\circ}, \partial S_{\circ}\right) \cong \Pi_{P} \rtimes \Gamma\left(S_{\circ, P}, \partial S_{\circ, P}\right) .
$$

Theorem 5.2. Let $\check{\Pi}_{P}$ be the closure of the fundamental group $\Pi_{P}$ in the $\mathscr{C}$-congruence completion $\check{\Gamma}\left(S_{\circ}, \partial S_{\circ}\right)^{\mathscr{C}}$. Then, the above decomposition of $\Gamma\left(S_{\circ}, \partial S_{\circ}\right)$ induces one:

$$
\check{\Gamma}\left(S_{\circ}, \partial S_{\circ}\right)^{\mathscr{C}} \cong \check{\Pi}_{P}^{\mathscr{C}} \rtimes \check{\Gamma}\left(S_{\circ, P}, \partial S_{\circ, P}\right)^{\mathscr{C}} .
$$

In particular, there is a split Birman short exact sequence:

$$
1 \rightarrow \check{\Pi}_{P}^{\mathscr{C}} \rightarrow \check{\Gamma}\left(S_{\circ}, \partial S_{\circ}\right)^{\mathscr{C}} \rightarrow \check{\Gamma}\left(S_{\circ, P}, \partial S_{\circ, P}\right)^{\mathscr{C}} \rightarrow 1
$$

Proof. The first remark is that, as a subgroup of $\Gamma\left(S_{\circ}\right)$, the group $s_{*}\left(\Gamma\left(S_{\circ, P}, \partial S_{\circ, P}\right)\right)$ is naturally isomorphic to the direct factor $\Gamma\left(s\left(S_{\circ, P}\right), \delta\right)$ of the stabilizer $\Gamma\left(S_{\circ}\right){ }_{\vec{\delta}}$. From Theorem 3.1, it then follows that the closure $\overline{s_{*}\left(\Gamma\left(S_{\circ, P}, \partial S_{\circ, P}\right)\right)}$ of the subgroup $s_{*}\left(\Gamma\left(S_{\circ, P}, \partial S_{\circ, P}\right)\right.$ in $\check{\Gamma}\left(S_{\circ}, \partial S_{\circ}\right)^{\mathscr{C}}$ is naturally isomorphic to the $\mathscr{C}$-congruence completion $\check{\Gamma}\left(S_{\circ}, P, \partial S_{\circ, P}\right)^{\mathscr{C}}$ of the mapping class group $\Gamma\left(S_{\circ, P}, \partial S_{\circ, P}\right)$ associated to the natural representation in $\operatorname{Aut}\left(\Pi_{P}\right)$ (cf. Definition 5.1).

Let us denote by $\tilde{\Gamma}\left(S_{\circ, P}, \partial S_{\circ, P}\right)^{\mathscr{C}}$ the profinite completion of $\Gamma\left(S_{\circ, P}, \partial S_{\circ, P}\right)$ induced by the $\mathscr{C}$-congruence completion $\check{\Gamma}\left(S_{\circ}, \partial S_{\circ}\right)^{\mathscr{C}}$ via the epimorphism $\Psi$ in the Birman exact sequence $(7)$ and by $\check{\Psi}^{\mathscr{C}}: \check{\Gamma}\left(S_{\circ}, \partial S_{\circ}\right)^{\mathscr{C}} \rightarrow \tilde{\Gamma}\left(S_{\circ, P}, \partial S_{\circ, P}\right)^{\mathscr{C}}$ the induced epimorphism. In order to prove the theorem, it is then enough to show that the restriction of $\check{\Psi}^{\mathscr{C}}$ to $\overline{s_{*}\left(\Gamma\left(S_{\circ, P}, \partial S_{\circ, P}\right)\right)}$ is an isomorphism.

Let $\widetilde{\Gamma}\left(S_{\circ, P}\right)^{\mathscr{C}}$ be the profinite completion of $\Gamma\left(S_{\circ, P}\right)$ induced by the $\mathscr{C}$-congruence completion $\check{\Gamma}\left(S_{\circ}\right)^{\mathscr{C}}$ via the natural epimorphism $\Gamma\left(S_{\circ}\right) \rightarrow \Gamma\left(S_{\circ, P}\right)$ (with kernel $\left.\Pi_{P}\right)$. By the various definitions involved, there is then a short exact sequence:

$$
1 \rightarrow \tau_{u}^{\hat{\mathbb{Z}}^{\mathscr{C}}} \rightarrow \tilde{\Gamma}\left(S_{\circ, P}, \partial S_{\circ, P}\right)^{\mathscr{C}} \rightarrow \tilde{\Gamma}\left(S_{\circ, P}\right)^{\mathscr{C}} \rightarrow 1
$$


After moding out the closure of $\Pi_{P}$ in both domain and codomain of the epimorphism $\hat{\Delta}_{P}^{\mathscr{C}}: \check{\Gamma}\left(S_{\circ}\right)^{\mathscr{C}} \rightarrow \check{\Gamma}\left(S_{\circ, P}, P\right)^{\mathscr{C}}$, we get an epimorphism $\bar{\Delta}_{P}^{\mathscr{C}}: \tilde{\Gamma}\left(S_{\circ, P}\right)^{\mathscr{C}} \rightarrow \check{\Gamma}\left(S_{\circ, P}\right)^{\mathscr{C}}$ and, after moding out the normal subgroup $\tau_{u}^{\hat{\mathbb{Z}}^{\mathscr{C}}}$ in both domain and codomain of the epimorphism $\check{\Psi}^{\mathscr{C}}: \overline{s_{*}\left(\Gamma\left(S_{\circ, P}, \partial S_{\circ, P}\right)\right)} \rightarrow \tilde{\Gamma}\left(S_{\circ, P}, \partial S_{\circ, P}\right)^{\mathscr{C}}$, we get an epimorphism $\check{\Gamma}\left(S_{\circ, P}\right)^{\mathscr{C}} \rightarrow \tilde{\Gamma}\left(S_{\circ, P}\right)^{\mathscr{C}}$ which, composed with $\bar{\Delta}_{P}^{\mathscr{C}}$, is an isomorphism and then is itself an isomorphism. Therefore, the restriction of $\check{\Psi}^{\mathscr{C}}$ to $\overline{s_{*}\left(\Gamma\left(S_{\circ, P}, \partial S_{\circ, P}\right)\right)}$ is an isomorphism as well.

By moding out the subgroup $\tau_{u}^{\hat{\mathbb{Z}}^{\mathscr{C}}}$ from the short exact sequence (8), we get:

Corollary 5.3. Let $\mathscr{C}$ be a class of finite groups and $S$ a nonclosed connected surface of negative Euler characteristic. Then, the natural epimorphism $\hat{\Delta}_{x}^{\mathscr{C}}: \check{\Gamma}\left(S_{x}\right)^{\mathscr{C}} \rightarrow \check{\Gamma}(S, x)^{\mathscr{C}}$ has the same kernel of the restriction $\left.\hat{\Delta}_{x}^{\mathscr{C}}\right|_{\Gamma^{\mathscr{C}}}$ and there is a short exact sequence:

$$
1 \rightarrow \check{\Pi}^{\mathscr{C}} \rightarrow \check{\Gamma}\left(S_{x}\right)^{\mathscr{C}} \rightarrow \check{\Gamma}(S)^{\mathscr{C}} \rightarrow 1
$$

\subsection{A $\mathscr{C}$-congruence subgroup property for genus 0 mapping class groups}

In the genus 0 case, the results of the previous section imply the weak version of the subgroup $\mathscr{C}$-congruence property, for $\mathscr{C}$ an arbitrary class of finite groups, and the standard version for $\mathscr{C}=\mathscr{F}$ or $(p)$ (cf. Definition 1.2):

Corollary 5.4. Let $\mathscr{C}$ be a class of finite groups, $S$ a connected surface of genus 0 and negative Euler characteristic and $x$ a point on $S$.

(i) There are natural epimorphism $\check{\Gamma}\left(S_{x}\right)^{\mathscr{C}} \rightarrow \widehat{\Gamma}\left(S_{x}\right)^{\mathscr{C}}$ and $\check{\Gamma}(S, x)^{\mathscr{C}} \rightarrow \widehat{\Gamma}(S, x)^{\mathscr{C}}$.

(ii) Let $\mathscr{C}$ be such that $\check{\Pi}^{\mathscr{C}} \equiv \widehat{\Pi}^{\mathscr{C}}$ for all surfaces $S$ of genus 0 (e.g. $\mathscr{C}=\mathscr{F}$ or $(p)$ ). Then, we have $\check{\Gamma}\left(S_{x}\right)^{\mathscr{C}} \equiv \widehat{\Gamma}\left(S_{x}\right)^{\mathscr{C}} \equiv \check{\Gamma}(S, x)^{\mathscr{C}} \equiv \widehat{\Gamma}(S, x)^{\mathscr{C}}$.

Proof. By the Birman short exact sequences (1) and (9), there is a commutative diagram with exact rows:

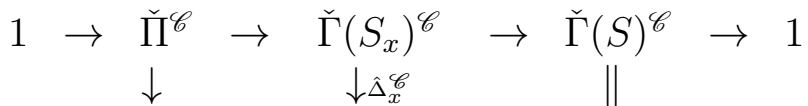

$$
\begin{aligned}
& 1 \rightarrow \widehat{\Pi}^{\mathscr{C}} \rightarrow \check{\Gamma}(S, x)^{\mathscr{C}} \rightarrow \check{\Gamma}(S)^{\mathscr{C}} \rightarrow 1,
\end{aligned}
$$

where all vertical maps are surjective. Induction on $-\chi(S)$, starting with $S$ a 3-punctured sphere $(-\chi(S)=1)$, for which $\Gamma(S)=\{1\}$, then yields both items of the corollary.

Remark 5.5. For $\mathscr{C}=\mathscr{F}$ and $\mathscr{C}=(p)$, item (ii) of Corollary 5.4 was already proved by Asada in [2]. 


\section{$6 \quad \mathscr{C}$-congruence subgroup properties for hyperelliptic mapping class groups}

The proof of Theorem 1.4 is based on the interpretation of hyperelliptic mapping class groups as fundamental groups of moduli stacks of complex hyperelliptic curves. Let us recall a few facts from Section 3 of [4]. The moduli stack of $n$-pointed, genus $g$ smooth hyperelliptic curves $\mathcal{H}_{g, n}$ can be described in terms of moduli of pointed genus 0 curves. Indeed, there is a natural $\mathbb{Z} / 2$-gerbe $\mathcal{H}_{g} \rightarrow \mathcal{M}_{0,[2 g+2]}$, for $g \geq 2$, defined assigning, to a genus $g$ hyperelliptic curve $C$, the genus zero quotient curve $C / \iota$ marked by the branch locus of the finite morphism $C \rightarrow C / \iota$, where $\iota$, as usual, denotes the hyperelliptic involution. Similarly, in the genus 1 case, there is a $\mathbb{Z} / 2$-gerbe $\mathcal{M}_{1,1} \rightarrow \mathcal{M}_{0,1[3]}$, where, by the notation "1[3]", we mean that one label is distinguished from the others (which instead are unordered).

For $2 g-2+n>0$, there is also a natural representable morphism $\mathcal{H}_{g, n+1} \rightarrow \mathcal{H}_{g, n}$, forgetting the $(n+1)$-th marked point, which is isomorphic to the universal $n$-punctured curve over $\mathcal{H}_{g, n}$. Thus, the fibre above an arbitrary closed point $x \in \mathcal{H}_{g, n}$ is diffeomorphic to $S_{g, n}$ and its fundamental group is isomorphic to $\Pi_{g, n}$. These morphisms induce, on topological fundamental groups, short exact sequences, for $g \geq 2$ :

$$
1 \rightarrow \mathbb{Z} / 2 \rightarrow \Upsilon_{g} \rightarrow \Gamma_{0,[2 g+2]} \rightarrow 1 \quad \text { and } \quad 1 \rightarrow \Pi_{g, n} \rightarrow \Upsilon_{g, n+1} \rightarrow \Upsilon_{g, n} \rightarrow 1
$$

For the algebraic fundamental groups, there are then short exact sequences (here and in the sequel, in order to simplify the notations, we omit to mention base points):

$$
1 \rightarrow \mathbb{Z} / 2 \rightarrow \pi_{1}^{\mathrm{alg}}\left(\mathcal{H}_{g}\right) \rightarrow \pi_{1}^{\mathrm{alg}}\left(\mathcal{M}_{0,[2 g+2]}\right) \rightarrow 1 \text { and } 1 \rightarrow \widehat{\Pi}_{g, n} \rightarrow \pi_{1}^{\mathrm{alg}}\left(\mathcal{H}_{g, n+1}\right) \rightarrow \pi_{1}^{\mathrm{alg}}\left(\mathcal{H}_{g, n}\right) \rightarrow 1
$$

where, for all $n \geq 0$, the groups $\pi_{1}^{\text {alg }}\left(\mathcal{H}_{g, n}\right)$ and $\pi_{1}^{\text {alg }}\left(\mathcal{M}_{0,[2 g+2]}\right)$ identify, respectively, with the profinite completions $\widehat{\Upsilon}_{g, n}$ of $\Upsilon_{g, n}$ and $\widehat{\Gamma}_{0,[2 g+2]}$ of $\Gamma_{0,[2 g+2]}$. For $g=1$, we just need to replace the short exact sequences which appear on the left in (10) and (11) above with $1 \rightarrow \mathbb{Z} / 2 \rightarrow \Gamma_{1,1} \rightarrow \Gamma_{0,1[3]} \rightarrow 1$ and $1 \rightarrow \mathbb{Z} / 2 \rightarrow \pi_{1}^{\text {alg }}\left(\mathcal{M}_{1,1}\right) \rightarrow \pi_{1}^{\text {alg }}\left(\mathcal{M}_{0,1[3]}\right) \rightarrow 1$, respectively.

The outer representation $\hat{\varrho}_{g, n}: \pi_{1}^{\text {alg }}\left(\mathcal{H}_{g, n}\right) \rightarrow \operatorname{Out}\left(\widehat{\Pi}_{g, n}\right)$, associated to the left short exact sequence in (11), is the algebraic monodromy representation of the punctured universal curve over $\mathcal{H}_{g, n}$. The $\mathscr{F}$-congruence subgroup property for the hyperelliptic mapping class group $\Upsilon_{g, n}$ is then equivalent to the faithfulness of $\hat{\varrho}_{g, n}$ (cf. [4]).

For $\mathscr{C}$ a class of finite groups, the kernel of the natural epimorphism $\widehat{\Pi}_{g, n} \rightarrow \widehat{\Pi}_{g, n}^{\mathscr{C}}$ is characteristic. Therefore, the representation $\hat{\varrho}_{g, n}$ induces a representation:

$$
\hat{\varrho}_{g, n}^{\mathscr{C}}: \pi_{1}^{\mathrm{alg}}\left(\mathcal{H}_{g, n}\right) \rightarrow \operatorname{Out}\left(\widehat{\Pi}_{g, n}^{\mathscr{C}}\right),
$$

which we call the universal hyperelliptic pro- $\mathscr{C}$ monodromy representation. The image of this representation is isomorphic to the $\mathscr{C}$-congruence completion $\check{\Upsilon}_{g, n}^{\mathscr{C}}$. Its kernel then identifies with the intersection $\cap_{\lambda \in \Lambda} U_{\lambda}$ of all $\mathscr{C}$-congruence open subgroups of $\widehat{\Upsilon}_{g, n} \cong$ $\pi_{1}^{\text {alg }}\left(\mathcal{H}_{g, n}\right)$. In particular, the kernel of $\hat{\varrho}_{g, n}^{\mathscr{C}}$ does not change if we restrict this representation to a $\mathscr{C}$-congruence open subgroup $U_{\lambda}$ of $\widehat{\Upsilon}_{g, n}$. 
For $\mathbb{Z} / 2 \in \mathscr{C}$, the profinite completion $\widehat{\Upsilon}(2)$ of the abelian level $\Upsilon(2)$ of $\Upsilon_{g, n}$ is a $\mathscr{C}$-congruence open subgroup of $\widehat{\Upsilon}_{g, n}$. Let then $\mathscr{K}_{g, n}^{\mathscr{C}}$ be the (characteristic) kernel of the natural epimorphism from the profinite completion to the pro- $\mathscr{C}$ completion $\widehat{\Upsilon}(2) \rightarrow \widehat{\Upsilon}(2)^{\mathscr{C}}$.

Item (i) of Theorem 1.4 is equivalent to the statement that, if $\mathbb{Z} / 2 \in \mathscr{C}$, the epimorphism $\widehat{\Upsilon}(2) \rightarrow \widehat{\Upsilon}(2)^{\mathscr{C}}$ factors through the representation $\hat{\varrho}_{g, n}^{\mathscr{C}}$ and an epimorphism:

$$
\check{\Upsilon}(2)^{\mathscr{C}} \rightarrow \widehat{\Upsilon}(2)^{\mathscr{C}} \quad \text { or equivalently } \quad \text { ker } \hat{\varrho}_{g, n}^{\mathscr{C}} \subseteq \mathscr{K}_{g, n}^{\mathscr{C}}
$$

Item (ii) of Theorem 1.4 is equivalent to the statement that, under the given hypothesis, the epimorphism $\widehat{\Upsilon}(2) \rightarrow \widehat{\Upsilon}(2)^{\mathscr{C}}$ factors through the representation $\hat{\varrho}_{g, n}^{\mathscr{C}}$ and an isomorphism:

$$
\check{\Upsilon}(2)^{\mathscr{C}} \stackrel{\sim}{\rightarrow} \widehat{\Upsilon}(2)^{\mathscr{C}} \quad \text { or equivalently } \quad \text { ker } \hat{\varrho}_{g, n}^{\mathscr{C}}=\mathscr{K}_{g, n}^{\mathscr{C}} \text {. }
$$

The étale covering $\mathcal{H}^{\lambda} \rightarrow \mathcal{H}_{g, n}$ associated to a (geometric) level $\Upsilon^{\lambda}$ of $\Upsilon_{g, n}$ is called a (geometric) level structure over $\mathcal{H}_{g, n}$. The abelian level structure $\mathcal{H}^{(m)}$ of order $m$, for $m \geq 2$, is the covering associated to the abelian level $\Upsilon(m)$ of $\Upsilon_{g, n}$. There is a standard procedure to simplify the structure of an algebraic stack $X$ by erasing a generic group of automorphisms $G$. The algebraic stack thus obtained is usually denoted by $X \rrbracket G$. The natural map $\mathcal{H}_{g} \rightarrow \mathcal{M}_{0,[2 g+2]}$ yields an isomorphism $\mathcal{H}_{g} \rrbracket\langle\iota\rangle \cong \mathcal{M}_{0,[2 g+2]}$. In Proposition 3.3 in [4], it was observed that the pull-back over $\mathcal{H}_{g}$ of the Galois étale covering $\mathcal{M}_{0,2 g+2} \rightarrow \mathcal{M}_{0,[2 g+2]}$ is the abelian level structure $\mathcal{H}_{g}^{(2)}$. More precisely:

Proposition 6.1. For $g \geq 2$, there is a natural isomorphism $\mathcal{H}^{(2)} \rrbracket\langle\iota\rangle \cong \mathcal{M}_{0,2 g+2}$. In particular, the abelian level of order 2 of $\Upsilon_{g}$ is described by the short exact sequence:

$$
1 \rightarrow\langle\iota\rangle \rightarrow \Upsilon(2) \rightarrow \Gamma_{0,2 g+2} \rightarrow 1
$$

Remark 6.2. Similarly, for the abelian level structure $\mathcal{M}^{(2)}$ over $\mathcal{M}_{1,1}$, there is a natural isomorphism $\mathcal{M}^{(2)} \rrbracket\langle\iota\rangle \cong \mathcal{M}_{0,4}$, where $\iota$ here denotes the generic elliptic involution and a short exact sequence $1 \rightarrow\langle\iota\rangle \rightarrow \Gamma(2) \rightarrow \Gamma_{0,4} \rightarrow 1$. From now on, for simplicity of exposition, we will focus on the moduli spaces of hyperelliptic curves $\mathcal{H}_{g, n}$, for $g \geq 2$, and leave to the reader the obvious reformulation and proof of the similar statements for the case $g=1$ and $n \geq 1$.

Let $\mathscr{C}_{g} \rightarrow \mathcal{H}_{g}$, for $g \geq 2$, be the universal curve. Removing Weierstrass points from its fibres, we obtain a $(2 g+2)$-punctured, genus $g$ curve $\mathscr{C}_{0} \rightarrow \mathcal{H}_{g}$. Let $C_{0}$ be the fiber of $\mathscr{C}_{0} \rightarrow \mathcal{H}_{g}$ over the base point of the algebraic fundamental group $\widehat{\Upsilon}_{g}$ of $\mathcal{H}_{g}$ and let us denote by

$$
\hat{\varrho}_{0}^{\mathscr{C}}: \widehat{\Upsilon}_{g} \hookrightarrow \operatorname{Out}\left(\hat{\pi}_{1}\left(C_{0}\right)^{\mathscr{C}}\right)
$$

the associated algebraic monodromy representation.

By Proposition 6.1, since $\iota \notin \Upsilon(4)$, there is a natural representable étale Galois morphism $\mathcal{H}^{(4)} \rightarrow \mathcal{M}_{0,2 g+2}$. Let $\mathscr{C}_{0}^{(4)} \rightarrow \mathcal{H}^{(4)}$ and $\mathcal{R}^{(4)} \rightarrow \mathcal{H}^{(4)}$ be, respectively, the pull-backs 
of the curve $\mathscr{C}_{0} \rightarrow \mathcal{H}_{g}$ and of the universal $(2 g+2)$-punctured, genus 0 curve $\mathcal{R} \rightarrow \mathcal{M}_{2 g+2}$ over $\mathcal{H}^{(4)}$. There is then a commutative diagram:

$$
\begin{array}{rlr}
\mathscr{C}_{0}^{(4)} & \stackrel{\psi}{\rightarrow} \mathcal{R}^{(4)} \\
& \searrow & \downarrow \\
& \mathcal{H}^{(4)},
\end{array}
$$

where $\psi$ is the étale, degree 2 map which, fibrewise, is the natural map to the quotient by the hyperelliptic involution. Pick a base point of $\mathcal{H}^{(4)}$ so that the fiber over it of the curve $\mathscr{C}_{0}^{(4)} \rightarrow \mathcal{H}^{(4)}$ identifies with $C_{0}$ and let $R$ be the fiber over the chosen base point of the curve $\mathcal{R}^{(4)} \rightarrow \mathcal{H}^{(4)}$. From the above diagram, we then get the algebraic monodromy representations: $\widehat{\Upsilon}(4) \rightarrow \operatorname{Out}\left(\hat{\pi}_{1}\left(C_{0}\right)^{\mathscr{C}}\right)$, which is just the restriction of $\hat{\varrho}_{0}^{\mathscr{C}}$ to $\widehat{\Upsilon}(4)$, has its same kernel and we denote in the same way, and $\hat{\varrho}_{\mathcal{R}}^{\mathscr{C}}: \widehat{\Upsilon}(4) \rightarrow \operatorname{Out}\left(\hat{\pi}_{1}(R)^{\mathscr{C}}\right)$. We have:

Lemma 6.3. If $\mathbb{Z} / 2 \in \mathscr{C}$, the representation $\hat{\varrho}_{0}^{\mathscr{C}}$ (13) has the same kernel of $\hat{\varrho}_{\mathcal{R}}$ so that the short exact sequence (12) induces one

$$
1 \rightarrow\langle\iota\rangle \rightarrow \hat{\varrho}_{0}^{\mathscr{C}}(\widehat{\Upsilon}(2)) \rightarrow \check{\Gamma}_{0,2 g+2}^{\mathscr{C}} \rightarrow 1
$$

Moreover, we have:

(i) $\operatorname{ker} \hat{\varrho}_{0}^{\mathscr{C}} \subseteq \mathscr{K}_{g}^{\mathscr{C}}$.

(ii) If the hypothesis of (ii) of Theorem 1.4 are satisfied, then ker $\hat{\varrho}_{0}^{\mathscr{C}}=\mathscr{K}_{g}^{\mathscr{C}}$.

Proof. Before we start the proof of the lemma, let us remark that the subgroup $\mathscr{K}_{g}^{\mathscr{C}}$ of $\widehat{\Upsilon}(2)$ is contained in any $\mathscr{C}$-open subgroup of this group and thus, in particular, is contained in $\widehat{\Upsilon}(4)$. In fact, the level $\Upsilon(4)$ has index a power of 2 in $\Upsilon(2)$ and hence its profinite completion $\widehat{\Upsilon}(4)$ is a $\mathscr{C}$-open normal subgroup of $\widehat{\Upsilon}(2)$.

Since $\mathbb{Z} / 2 \in \mathscr{C}$, the group $\hat{\pi}_{1}\left(C_{0}\right)^{\mathscr{C}}$ identifies with an open normal subgroup of $\hat{\pi}_{1}(R)^{\mathscr{C}}$ of index 2. The inclusion ker $\hat{\varrho}_{0}^{\mathscr{C}} \subseteq$ ker $\hat{\varrho}_{\mathcal{R}}^{\mathscr{C}}$ then follows from:

Lemma 6.4. Let $\widehat{F}^{\mathscr{C}}$ be a free pro-C्C group of finite rank and $N$ an open normal subgroup of $\widehat{F}^{\mathscr{C}}$. If $\alpha$ is an automorphism of $\widehat{F}^{\mathscr{C}}$ which restricts to the identity on $N$, then $\alpha$ is the identity automorphism.

Proof. The proof is essentially the same as the proof of Lemma 8 in [2]. The only difference is that, where Asada appeals to his identity (1.3.1), we need to use Lemma 2.3.

To see the other inclusion ker $\hat{\varrho}_{\mathcal{R}}^{\mathscr{C}} \subseteq$ ker $\hat{\varrho}_{0}^{\mathscr{C}}$, let us observe that, if $f \in \operatorname{ker} \hat{\varrho}_{\mathcal{R}}^{\mathscr{C}}$, the element $\hat{\varrho}_{0}^{\mathscr{C}}(f)$ acts on $\hat{\pi}_{1}\left(C_{0}\right)^{\mathscr{C}}$ through a power of the hyperelliptic involution. But then $\hat{\varrho}_{0}^{\mathscr{C}}(f)=1$, since $\hat{\varrho}_{0}^{\mathscr{C}}(f)$ acts trivially on $H_{1}\left(C_{0}, \mathbb{Z} / 4\right)$ while the hyperelliptic involution acts on $H_{1}\left(C_{0}, \mathbb{Z} / 4\right)$ by multiplication by -1 .

Now, since $\iota \notin \widehat{\Upsilon}(4)$, the natural epimorphism $\widehat{\Upsilon}(2) \rightarrow \widehat{\Gamma}_{2 g+2,0}$ identifies $\widehat{\Upsilon}(4)$ with a $\mathscr{C}$-open subgroup of $\widehat{\Gamma}_{2 g+2,0}$ and then the subgroup $\mathscr{K}_{g}^{\mathscr{C}}$ of $\widehat{\Upsilon}(4)$ is identified with the kernel of the natural epimorphism $\widehat{\Gamma}_{2 g+2,0} \rightarrow \widehat{\Gamma}_{2 g+2,0}^{\mathscr{C}}$. The short exact sequence (14) and items (i), (ii) of the lemma now follow from Corollary 5.4, Proposition 6.1 and the part of the lemma which we already proved. 
We can now prove a special case of Theorem 1.4:

Lemma 6.5. For $\mathscr{C}$ a class of finite groups such that $\mathbb{Z} / 2 \in \mathscr{C}$, we have:

(i) $\operatorname{ker} \hat{\varrho}_{g, 2 g+2}^{\mathscr{C}} \subseteq \mathscr{K}_{g, 2 g+2}^{\mathscr{C}}$.

(ii) If the hypothesis of (ii) of Theorem 1.4 are satisfied, then ker $\hat{\varrho}_{g, 2 g+2}^{\mathscr{C}}=\mathscr{K}_{g, 2 g+2}^{\mathscr{C}}$.

Proof. The universal curve $\mathscr{C}^{(2)} \rightarrow \mathcal{H}_{g}^{(2)}$ over the abelian level structure of order 2 is endowed with $2 g+2$ sections corresponding to the Weierstrass points on the fibres. Let us fix an order on this set of sections. By the universal property of the level structure $\mathcal{H}_{g, n}^{(2)}$, there is then a morphism $s: \mathcal{H}_{g}^{(2)} \rightarrow \mathcal{H}_{g, 2 g+2}^{(2)}$, which is a section of the natural projection $p: \mathcal{H}_{g, 2 g+2}^{(2)} \rightarrow \mathcal{H}_{g}^{(2)}$ (forgetting the labels). The morphism $p$ is smooth and its fibre above a closed point $[C] \in \mathcal{H}_{g}$ is the configuration space of $2 g+2$ points on the curve $C$.

Let us denote by $S_{g}(n)$ the configuration space of $n$ points on the closed Riemann surface $S_{g}$ and by $\Pi_{g}(n)$ its fundamental group. All fibres of $p$ above closed points of $\mathcal{H}_{g}$ are diffeomorphic to $S_{g}(2 g+2)$. Therefore, we have a semidirect product decomposition of the fundamental group $\Upsilon_{g, 2 g+2}(2)$ of $\mathcal{H}_{g, 2 g+2}^{(2)}$ :

$$
\Upsilon_{g, 2 g+2}(2)=\Pi_{g}(2 g+2) \rtimes \Upsilon_{g}(2) .
$$

In Section 3 of [4], we saw that this decomposition induces one:

$$
\widehat{\Upsilon}_{g, 2 g+2}(2) \cong \widehat{\Pi}_{g}(2 g+2) \rtimes \widehat{\Upsilon}_{g}(2) .
$$

For $n \geq 1$, let us denote by $\check{\Pi}_{g}(2 g+2)^{\mathscr{C}}$ the closure of $\Pi_{g}(2 g+2)$ in the $\mathscr{C}$-congruence completion $\check{\Upsilon}_{g, 2 g+2}^{\mathscr{C}}$. By Corollary 5.3, for all $n \geq 1$, there is then a short exact sequence:

$$
1 \rightarrow \check{\Pi}_{g, n}^{\mathscr{C}} \rightarrow \check{\Pi}_{g}(n+1)^{\mathscr{C}} \rightarrow \check{\Pi}_{g}(n)^{\mathscr{C}} \rightarrow 1 \text {. }
$$

Hence, by induction on $n$ and (i) of Proposition 2.4, we see that, for all $n \geq 1$, there is a natural epimorphism $\check{\Pi}_{g}(n)^{\mathscr{C}} \rightarrow \widehat{\Pi}_{g}(n)^{\mathscr{C}}$.

By Lemma 6.3, in order to prove Lemma 6.5, it is then enough to show that the $\mathscr{C}$ congruence completion of $\Upsilon_{g, 2 g+2}(2)$ admits a semidirect product decomposition:

$$
\check{\Upsilon}_{g, 2 g+2}(2)^{\mathscr{C}} \cong \check{\Pi}_{g}(2 g+2)^{\mathscr{C}} \rtimes \hat{\varrho}_{0}^{\mathscr{C}}\left(\widehat{\Upsilon}_{g}(2)\right)
$$

To prove the existence of the decomposition (16), we have to show that $\check{\Pi}_{g}(2 g+2)^{\mathscr{C}}$ has trivial intersection with $\hat{\varrho}_{0}^{\mathscr{C}}\left(\widehat{\Upsilon}_{g}(2)\right)$ inside the pro- $\mathscr{C}$ congruence hyperelliptic mapping class group $\check{\Upsilon}_{g, 2 g+2}(2)^{\mathscr{C}}$.

As in the proof of Lemma 3.7 in [4], the key observation is that the group $\hat{\varrho}_{0}^{\mathscr{C}}\left(\widehat{\Upsilon}_{g}(2)\right)$ centralizes the hyperelliptic involution $\hat{\varrho}_{0}^{\mathscr{C}}(\iota)$, which we simply denote by $\iota$ (as well all the images of this element in the groups $\check{\Upsilon}_{g, n}(2)^{\mathscr{C}}$, for $\left.0 \leq n<2 g+2\right)$. To conclude, we then have to show that instead the centralizer of $\iota$ in $\check{\Pi}_{g}(2 g+2)^{\mathscr{C}}$ is trivial. By the short exact sequence (15) and induction on $n$, this last claim is implied by the lemma: 
Lemma 6.6. For all $0 \leq n \leq 2 g+1$ (where, for $g=1$, we let $n \geq 1$ ), the subgroup $\langle\iota\rangle$ is self-normalizing in $\check{\Pi}_{g, n}^{\mathscr{C}} \cdot\langle\iota\rangle$.

Proof. The subgroup $\Pi_{g, n} \cdot\langle\iota\rangle$ of the hyperelliptic mapping class group $\Upsilon_{g, n+1}$ identifies with the fundamental group of the orbifold quotient by the hyperelliptic involution of the punctured hyperelliptic Riemann surface obtained from a closed hyperelliptic Riemann surface $\left(S_{g}, \iota\right)$ of genus $g$ removing $n$ of its $2 g+2$ Weierstrass points.

The first remark is then that we can reduce to the case $n=0$. In fact, $\Pi_{g, n}$ identifies with a subgroup of the fundamental group $\pi_{1}(S, x)$ of the closed hyperelliptic Riemann surface $S$ of genus $g+n$ obtained from $S_{g, n}$ capping each of the $n$ punctures with a surface of genus 1, where we let $x \in S_{g, n} \subset S$ be one of the Weierstrass points of $S_{g}$ which has not been removed. An immediate consequence of Theorem 3.1 is then that the closure of $\Pi_{g, n}$ in the $\mathscr{C}$-congruence completion $\check{\Gamma}\left(S_{x}\right)^{\mathscr{C}}$ is $\check{\Pi}_{g, n}^{\mathscr{C}}$, where we identify $\pi_{1}(S, x)$ with the kernel of the natural epimorphism $\Gamma\left(S_{x}\right) \rightarrow \Gamma(S)$ and $\Pi_{g, n}$ with a subgroup of $\pi_{1}(S, x)$ as explained above. For $n=0$, Lemma 6.6 and then Lemma 6.5 follow from the lemma:

Lemma 6.7. Let $[S / \iota]$ be the orbifold quotient of a hyperelliptic closed Riemann surface $S$ by the hyperelliptic involution $\iota$ and $\pi: S_{N} \rightarrow[S / \iota]$ the unramified covering associated to a finite index normal proper subgroup $N$ of $\Pi:=\pi_{1}(S, x)$ preserved by $\iota$, so that the covering transformation group of $\pi$ is naturally isomorphic to $G:=G_{N} \rtimes\langle\iota\rangle$, where $G_{N}:=\Pi / N$. Then, $S_{N}$ is not hyperelliptic and $\iota$ is self-centralizing in $G$.

Proof. We will show that the lemma is a consequence of the representation theory of the $G$-module $H^{1}\left(S_{N}, \mathbb{C}\right)$. By restriction, this is also a $G_{N}$-module and its $G$-module and $G_{N}$-module structures are related in the following way (cf. Proposition 5.1 in [10]). Let $V \neq\{0\}$ be an irreducible $G$-submodule of $H^{1}\left(S_{N}, \mathbb{C}\right)$, there are then two possibilities:

(i) $V$ is also irreducible as $G_{N}$-module and $\iota$ acts on it either trivially or by multiplication by -1 ;

(ii) $V$ is the sum of two irreducible and not isomorphic $G_{N^{-}}$submodules $W$ and $\iota(W)$.

Moreover, every irreducible $G$-submodule of $H^{1}\left(S_{N}, \mathbb{C}\right)$ is of one of the two above types. Let us show that the first possibility (i) occurs only when $V$ is a trivial $G_{N}$-submodule of $H^{1}\left(S_{N}, \mathbb{C}\right)$ on which $\iota$ acts by multiplication by -1 .

Let us consider first the case when $\iota$ acts trivially on $V$ and then when it acts by multiplication by -1 . Let $Q_{1}, \ldots, Q_{2 g+2}$ be the branch points of the covering $\pi: S_{N} \rightarrow$ $S / G$ and $G_{P_{i}}$ the corresponding stabilizer of a ramification point $P_{i}$ lying over $Q_{i}$, for $i=1, \ldots, 2 g+2$, where $g \geq 2$ is the genus of $S$. These are cyclic groups of order 2 generated by some conjugate of $\iota$. If $V$ is a nontrivial $G$-module, its multiplicity $\mu$ in $H^{1}\left(S_{N}, \mathbb{C}\right.$ ) is given by the formula (see, for instance, Corollary to Theorem 2 and formula (5) in Section 1 of [16], but the formula can also be proven with elementary methods):

$$
\mu=2 \operatorname{dim} V\left(g\left(S_{N} / N\right)-1\right)+\sum_{i=1}^{2 g+2}\left(\operatorname{dim} V-\operatorname{dim} V^{G_{P_{i}}}\right)
$$


where $g\left(S_{N} / N\right)$ is the genus of the quotient $S_{N} / N$. If the $G$-module $V$ is trivial, its multiplicity, of course, is simply $\mu=2 g\left(S_{N} / N\right)$. Therefore, in case $\iota$ acts trivially on $V$, we have that $\operatorname{dim} V-\operatorname{dim} V^{\iota}=0$. By the above formula, since $g\left(S_{N} / N\right)=0$, the multiplicity of the irreducible $G$-module $V$ in $H^{1}\left(S_{N}, \mathbb{C}\right)$ is then $-2 \operatorname{dim} V$, an absurd.

Let us then consider the case when $\iota$ acts by multiplication by -1 on $V$. We have that $\operatorname{dim} V-\operatorname{dim} V^{\iota}=\operatorname{dim} V$, which, by the same formula, implies that the irreducible $G$-module $V$ has multiplicity $2 g \cdot \operatorname{dim} V$ in $H^{1}\left(S_{N}, \mathbb{C}\right)$. However, by our assumptions, the group $G_{N}$ operates freely on $S_{N}$. Hence, the same formula, applied to the covering $p_{N}: S_{N} \rightarrow S$, implies that, as a $G_{N}$-module, $H^{1}\left(S_{N}, \mathbb{C}\right)$ consists of $2 g-2$ copies of the regular representation plus two copies of the trivial $G_{N}$-module. Therefore, $\iota$ acts by multiplication by -1 exactly when $V$ is the trivial $G_{N}$-module, i.e. $V \subset H^{1}\left(S_{N} / G_{N}, \mathbb{C}\right)$. This also shows that $S_{N}$ is not hyperelliptic and, in particular, $H^{1}\left(S_{N}, \mathbb{C}\right)^{\iota} \neq\{0\}$.

Let us now suppose that $\alpha \neq 1$ is an element of $G_{N}$ which commutes with $\iota$ and let $C_{\alpha}$ the cyclic subgroup of $G$ generated by $\alpha$. Then $H^{1}\left(S_{N}, \mathbb{C}\right)^{\iota}$ is a $C_{\alpha}$-module and so it contains an irreducible $C_{\alpha}$-submodule $W \neq\{0\}$. Since $C_{\alpha} \subseteq G_{N}$, there is an irreducible $G_{N^{-}}$submodule $V$ of $H^{1}\left(S_{N}, \mathbb{C}\right)$ such that $W \subseteq V$. Now, $\iota(V) \cap V \supseteq W$. Therefore, from the above discussion, it follows that $\iota(V)=V$ and $\iota$ acts on it by multiplication by -1 , which cannot be because $V \supseteq W$. Thus, we conclude that $Z_{G}(\iota)=\langle\iota\rangle$.

Proof of Theorem 1.4. (i): By the Birman exact sequence (9), for $n \geq 1$, there is a commutative diagram with exact rows

$$
\begin{aligned}
& 1 \rightarrow \widehat{\Pi}_{g, n} \rightarrow \widehat{\Upsilon}_{g, n+1}(2) \rightarrow \widehat{\Upsilon}_{g, n}(2) \rightarrow 1
\end{aligned}
$$

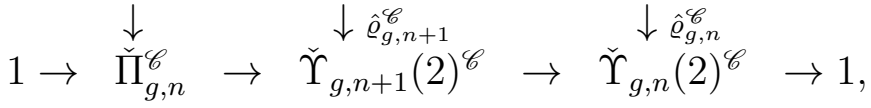

where the epimorphism $\widehat{\Pi}_{g, n} \rightarrow \widehat{\Pi}_{g, n}^{\mathscr{C}}$ factors through the epimorphism $\check{\Pi}_{g, n}^{\mathscr{C}} \rightarrow \widehat{\Pi}_{g, n}^{\mathscr{C}}$ (cf. (i) of Proposition 2.4). Proceeding by ascending and then descending induction on $n$, we see that the natural epimorphism $\widehat{\Upsilon}_{g, n}(2) \rightarrow \widehat{\Upsilon}_{g, n}(2)^{\mathscr{C}}$ factors through $\hat{\varrho}_{g, n}^{\mathscr{C}}$ and an epimorphism $\check{\Upsilon}_{g, n}(2)^{\mathscr{C}} \rightarrow \widehat{\Upsilon}_{g, n}(2)^{\mathscr{C}}$ both for $n \geq 2 g+2$ and for $1 \leq n \leq 2 g+2$.

(ii): The hypothesis $\check{\Pi}_{g, n}^{\mathscr{C}} \equiv \widehat{\Pi}_{g, n}^{\mathscr{C}}$ for all $n \geq 0$ and (ii) of Lemma 6.5 immediately imply that $\check{\Upsilon}_{g, n}(2)^{\mathscr{C}}$ is a pro- $\mathscr{C}$ group for all $n \geq 0$. Therefore, by the Birman exact sequence (3), there is a commutative diagram with exact rows:

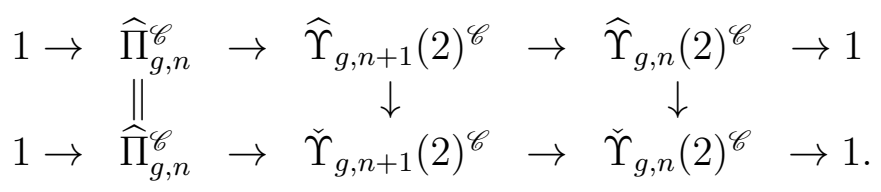

Proceeding by induction, we see that the natural epimorphism $\widehat{\Upsilon}_{g, n}(2)^{\mathscr{C}} \rightarrow \check{\Upsilon}_{g, n}(2)^{\mathscr{C}}$ is an isomorphism for both $n \geq 2 g+2$ and $0 \leq n \leq 2 g+2$. 


\section{Orbits of simple elements in a pro- $\mathscr{C}$ surface group}

In this section, we will prove a geometric version of a conjecture by Gelander and Lubotzky. Let us recall that an element of a free group is primitive, if it belongs to some minimal generating set. For $F$ a free group of finite rank, the conjecture states that the intersection with $F$ of the $\operatorname{Aut}(\hat{F})$-orbit of a primitive element of $F$ (where we identify $F$ with a subgroup of its profinite completion $\hat{F}$ ) consists of primitive elements. Otherwise stated, the set of primitive elements of $F$ is closed in the profinite topology. This conjecture has been proved by Puder and Parzanchevski (cf. Corollary 8.1 in [21]).

Shortly after the publication of [7], Pavel Zalesskii and I realized that (ii) of Theorem 4.3 and Proposition 4.1 of that paper imply a strong geometric version of the above conjecture for closed surface groups. For a surface group $\Pi=\pi_{1}(S, x)$ (where the surface $S$ is of finite type but not necessarily closed), the notion which roughly corresponds to that of primitive element is that of simple element. An element of $\Pi$ is simple if its free homotopy class contains a simple closed curve.

An immediate consequence of the aforementioned results is then that, for any class of finite groups $\mathscr{C}$, the set of simple elements of a closed surface group $\Pi$ is closed in the pro- $\mathscr{C}$ topology on $\Pi$. In this section, this result will be extended to any connected surface $S$ of negative Euler characteristic. Moreover, we will show that, for $\mathscr{C}=\mathscr{F}$ or $(p)$, the orbits of simple elements by $\mathscr{C}$-congruence subgroups of $\Gamma(S, x)$ are also closed for the pro- $\mathscr{C}$ topology on $\Pi$.

\subsection{The pro- $\mathscr{C}$ Reidemeister pairing}

In order to proceed, we need first to extend the results of Section 4 in [8] to an arbitrary surface group. Let, as usual, $S$ be a surface of finite negative Euler characteristic and let $\Pi$ be its fundamental group. It is then clear how to define a pro- $\mathscr{C}$ Reidemeister pairing:

$$
\mathfrak{R}_{\mathscr{C}}: \widehat{\Pi}^{\mathscr{C}} \times \widehat{\Pi}^{\mathscr{C}} \rightarrow \mathbb{Q}_{p}\left[\left[\widehat{\Pi}^{\mathscr{C}}\right]\right],
$$

which is continuous and invariant under the natural action by conjugation of the group $\widehat{\Pi}^{\mathscr{C}}$ on the domain. We just need to follow step by step the definitions given in Section 4 of [8], where, for a normal $\mathscr{C}$-open subgroup $K$ of $\widehat{\Pi}^{\mathscr{C}}$, we replace the homology group $H_{1}\left(K, \mathbb{Q}_{p}\right)$ with the homology group $H_{1}\left(\bar{S}_{K}, \mathbb{Q}_{p}\right)$ of the closed surface $\bar{S}_{K}$ associated to $K$ (cf. Section 4.1). As in [8], Theorem 2.3 ibid. then implies:

Theorem 7.1. Let $\mathscr{C}$ be a class of finite groups, $S$ a surface of negative Euler characteristic and let $\Pi=\pi_{1}(S, x)$ for some base point $x \in S$.

(i) The free homotopy classes of a pair of elements $\alpha, \beta \in \Pi$ contain disjoint representatives if and only if $\Re_{\mathscr{C}}(\alpha, \beta)=0$.

(ii) The free homotopy class of a nonpower element $\gamma \in \Pi$ contains a simple closed curve if and only if $\mathfrak{R}_{\mathscr{C}}(\gamma, \gamma)=0$. 


\subsection{The set of simple elements is closed in the pro- $\mathscr{C}$ topology}

In order to extend Proposition 4.1 [8] to an arbitrary surface group $\Pi$, we have to restrict the group of automorphisms of $\widehat{\Pi}^{\mathscr{C}}$ to those which are meaningful from a geometric point of view:

Definition 7.2. For every characteristic $\mathscr{C}$-open subgroup $K$ of $\widehat{\Pi}^{\mathscr{C}}$, let $N_{K}$ be the kernel of the natural epimorphism $\hat{\pi}_{1}\left(S_{K}, \tilde{x}\right)^{\mathscr{C}} \rightarrow \hat{\pi}_{1}\left(\bar{S}_{K}, \tilde{x}\right)^{\mathscr{C}}$, where $\tilde{x} \in S_{K}$ is a base point lying over the base point $x \in S$ of $\Pi$ and we identify $K$ with the pro- $\mathscr{C}$ completion $\hat{\pi}_{1}\left(S_{K}, \tilde{x}\right)^{\mathscr{C}}$ of the fundamental group $\pi_{1}\left(S_{K}, \tilde{x}\right)$. We say that an automorphism $f \in \operatorname{Aut}\left(\widehat{\Pi}^{\mathscr{C}}\right)$ is coherent if, for all subgroups $K$ of $\widehat{\Pi}^{\mathscr{C}}$ as above, $f$ preserves the subgroup $N_{K}$ (so that it induces an automorphism $\bar{f}_{K}$ of the quotient group $\left.\hat{\pi}_{1}\left(\bar{S}_{K}, \tilde{x}\right)^{\mathscr{C}}\right)$. Let us then denote by Aut ${ }^{\mathrm{coh}}\left(\widehat{\Pi}^{\mathscr{C}}\right)$ the subgroup of Aut $\left(\widehat{\Pi}^{\mathscr{C}}\right)$ consisting of coherent automorphisms.

Remark 7.3. Observe that $\mathrm{Aut} \mathrm{coh}^{\mathrm{coh}}\left(\widehat{\Pi}^{\mathscr{C}}\right)$ is a closed subgroup of $\operatorname{Aut}\left(\widehat{\Pi}^{\mathscr{C}}\right)$ which contains the image of the pro- $\mathscr{C}$ congruence mapping class group $\check{\Gamma}(S, x)^{\mathscr{C}}$ under the natural representation. Moreover, if we identify $\widehat{\Pi}^{\mathscr{C}}$ with the geometric pro- $\mathscr{C}$ algebraic fundamental group of a smooth projective curve $C$ defined over $\mathbb{Q}$ endowed with a rational point $x \in C$, Aut ${ }^{\text {coh }}\left(\widehat{\Pi}^{\mathscr{C}}\right)$ also contains the image of the absolute Galois group $G_{\mathbb{Q}}$ under the associated Galois representation.

The following, more familiar, characterization of coherent automorphisms is possibly of independent interest:

Proposition 7.4. Let $S=S_{g, n}$, for $2 g-2+n>0$, and denote by $u_{i} \in \Pi$ a simple loop around the puncture on $S$ obtained removing $P_{i}$ from $S_{g}$, for $i=1, \ldots, n$. Then we have:

$\operatorname{Aut}^{\operatorname{coh}}\left(\widehat{\Pi}^{\mathscr{C}}\right)=\left\{f \in \operatorname{Aut}\left(\widehat{\Pi}^{\mathscr{C}}\right) \mid f\left(u_{i}\right) \sim u_{\sigma(i)}^{s_{i}}\right.$ for some $\sigma \in \Sigma_{n}$ and $\left.s_{i} \in\left(\hat{\mathbb{Z}}^{\mathscr{C}}\right)^{*}, i=1, \ldots, n\right\}$.

Proof. This is a highly nontrivial but immediate consequence of Theorem 1.5 in [8].

The same proof (word by word) of Proposition 4.1 in [8] then gives:

Proposition 7.5. There is a character $\chi_{p}: \operatorname{Aut}^{\mathrm{coh}}\left(\widehat{\Pi}^{\mathscr{C}}\right) \rightarrow \mathbb{Z}_{p}^{*}$ such that, for all $f \in$ $\operatorname{Aut}^{\mathrm{coh}}\left(\widehat{\Pi}^{\mathscr{C}}\right)$ and $\alpha, \beta \in \widehat{\Pi}^{\mathscr{C}}$, we have $\mathfrak{R}_{\mathscr{C}}(f(\alpha), f(\beta))=\chi_{p}(f) \cdot \mathfrak{R}_{\mathscr{C}}(\alpha, \beta)$. For $f \in \operatorname{Inn}\left(\widehat{\Pi}^{\mathscr{C}}\right)$, we have $\chi_{p}(f)=1$ and so the character $\chi_{p}$ descends to a character $\bar{\chi}_{p}$ : Out ${ }^{\operatorname{coh}}\left(\widehat{\Pi}^{\mathscr{C}}\right) \rightarrow \mathbb{Z}_{p}^{*}$.

Let us observe that, by the various universal properties involved, we can extend the pro- $\mathscr{C}$ Reidemeister pairing to a bilinear pairing: $\mathbb{Q}_{p}\left[\left[\widehat{\Pi}^{\mathscr{C}}\right]\right] \otimes \mathbb{Q}_{p}\left[\left[\widehat{\Pi}^{\mathscr{C}}\right]\right] \rightarrow \mathbb{Q}_{p}\left[\left[\widehat{\Pi}^{\mathscr{C}}\right]\right]$, which is also continuous and invariant under the conjugacy action of $\widehat{\Pi}^{\mathscr{C}}$ on the domain.

On the $\mathbb{Q}_{p}$-vector space $\mathbb{Q}_{p}\left[\left[\widehat{\Pi}^{\mathscr{C}}\right]\right]$, there is a natural Aut ${ }^{\text {coh }}\left(\widehat{\Pi}^{\mathscr{C}}\right)$-module structure induced by the continuous action of $\operatorname{Aut}^{\operatorname{coh}}\left(\widehat{\Pi}^{\mathscr{C}}\right)$ on $\widehat{\Pi}^{\mathscr{C}}$. We let instead $\mathbb{Q}_{p}\left[\left[\widehat{\Pi}^{\mathscr{C}}\right]\right](1)$ be the Aut ${ }^{\mathrm{coh}}\left(\widehat{\Pi}^{\mathscr{C}}\right)$-module defined letting the group Aut ${ }^{\mathrm{coh}}\left(\widehat{\Pi}^{\mathscr{C}}\right)$ act on the $\mathbb{Q}_{p}$-vector space 
$\mathbb{Q}_{p}\left[\left[\widehat{\Pi}^{\mathscr{C}}\right]\right]$ through the character $\chi_{p}$. By Proposition 7.5 , with these definitions, the bilinear pairing just defined gives rise to an $\mathrm{Aut}^{\mathrm{coh}}\left(\widehat{\Pi}^{\mathscr{C}}\right)$-equivariant bilinear pairing:

$$
\mathfrak{R}_{\mathscr{C}}\left({ }_{-},{ }_{-}\right): \mathbb{Q}_{p}\left[\left[\widehat{\Pi}^{\mathscr{C}}\right]\right] \otimes \mathbb{Q}_{p}\left[\left[\widehat{\Pi}^{\mathscr{C}}\right]\right] \rightarrow \mathbb{Q}_{p}\left[\left[\widehat{\Pi}^{\mathscr{C}}\right]\right](1),
$$

which we call the pro- $\mathscr{C}$ Reidemeister bilinear pairing. Let us observe that, for $\mathscr{C}=(p)$, if we give $\mathbb{Q}_{p}\left[\left[\widehat{\Pi}^{(p)}\right]\right]$ the canonical (pro) mixed Hodge structure defined by Hain in $[11]$ and to $\mathbb{Q}_{p}\left[\left[\widehat{\Pi}^{\mathscr{C}}\right]\right](1)$ the Hodge structure of weight -2 obtained tensoring the trivial Hodge structure on $\mathbb{Q}_{p}\left[\left[\widehat{\Pi}^{(p)}\right]\right]$ by a Tate twist, the bilinear pairing $\mathfrak{R}_{p}\left({ }_{-},{ }_{-}\right)$becomes a morphism of (pro) mixed Hodge structures. These facts should be compared with Theorem 1 and Question 1.2 in [12] (cf. also Section 7.4).

Proposition 7.5 and Theorem 7.1 then immediately imply:

Corollary 7.6. For $\gamma \in \Pi$ a simple element, we have

$$
\operatorname{Aut}^{\mathrm{coh}}\left(\widehat{\Pi}^{\mathscr{C}}\right) \cdot \gamma \cap \Pi=\Gamma(S, x) \cdot \gamma
$$

where we identify $\Pi$ with a subgroup of its pro- $\mathscr{C}$ completion $\widehat{\Pi}^{\mathscr{C}}$. In particular, the set of simple elements of $\Pi$ is closed in the pro- $\mathscr{C}$ topology of $\Pi$.

\subsection{The $\mathscr{C}$-congruence orbit of a simple element $\left(\check{\Pi}^{\mathscr{C}} \equiv \widehat{\Pi}^{\mathscr{C}}\right)$}

In this section, we assume that $\check{\Pi}^{\mathscr{C}} \equiv \widehat{\Pi}^{\mathscr{C}}$ (e.g. $\mathscr{C}=\mathscr{F}$ or $\mathscr{C}=(p)$ ). In particular, by Corollary 4.5 and Corollary 4.10 , the $\mathscr{C}$-congruence topology on the mapping class group $\Gamma(S, x)$ is defined unambigously. We then have:

Corollary 7.7. Let $\mathscr{C}$ be a class of finite groups such that $\check{\Pi}^{\mathscr{C}} \equiv \widehat{\Pi}^{\mathscr{C}}$ (e.g. $\mathscr{C}=\mathscr{F}$ or $\mathscr{C}=(p)$ ) and let $U$ be an open subgroup of $\check{\Gamma}(S, x)^{\mathscr{C}}$ and $\gamma \in \Pi$ a simple element. Then:

$$
U \cdot \gamma \cap \Pi=(U \cap \Gamma(S, x)) \cdot \gamma
$$

where we identify $\Gamma(S, x)$ with a subgroup of $\check{\Gamma}(S, x)^{\mathscr{C}}$. In particular, orbits of simple elements by $\mathscr{C}$-congruence open subgroups of $\Gamma(S, x)$ are closed in the pro-C $\mathscr{C}$ topology of $\Pi$.

Proof. By Corollary 7.6, in order to prove Corollary 7.7, it is enough to show that the stabilizer $\check{\Gamma}_{\gamma}^{\mathscr{C}}$ of a simple element $\gamma \in \Pi \subset \widehat{\Pi}^{\mathscr{C}}$ for the action of the pro- $\mathscr{C}$ congruence mapping class group $\check{\Gamma}(S, x)^{\mathscr{C}}$ on $\widehat{\Pi}^{\mathscr{C}}$ is the closure of the stabilizer $\Gamma_{\gamma}$ of $\gamma$ for the action of $\Gamma(S, x)$ on $\Pi$. These stabilizer are, respectively, the centralizers in $\check{\Gamma}(S, x)^{\mathscr{C}}$ and $\Gamma(S, x)$ of the bounding pair map determined by $\gamma$ on $S$. The claim then follows from Corollary 4.3 and Theorem 3.6.

\subsection{Some open questions}

In Corollary 7.6, we have seen that the intersection of the $\operatorname{Aut}^{\mathrm{coh}}\left(\widehat{\Pi}^{\mathscr{C}}\right)$-orbit in $\widehat{\Pi}^{\mathscr{C}}$ of a simple element $\gamma \in \Pi$ with $\Pi$ coincides with the $\Gamma(S, x)$-orbit of $\gamma$. A natural question is then the following: 
Question 7.8. Does the closure in $\widehat{\Pi}^{\mathscr{C}}$ of the $\Gamma(S, x)$-orbit of a simple element $\gamma \in \Pi$ coincides with the Aut ${ }^{\mathrm{coh}}\left(\widehat{\Pi}^{\mathscr{C}}\right)$-orbit in $\widehat{\Pi}^{\mathscr{C}}$ of the same element?

An affirmative answer to this question would put strong restrictions on the profinite group $A \mathrm{At}^{\mathrm{coh}}\left(\widehat{\Pi}^{\mathscr{C}}\right)$ and I believe that it may have important implications for GrothendieckTeichmüller theory. Related to Question 7.8 is the problem of characterizing the set of simple elements in $\widehat{\Pi}^{\mathscr{C}}$, which we define to be the closure in $\widehat{\Pi}^{\mathscr{C}}$ of the set of simple elements of $\Pi$.

Let us observe that the simple elements of $\widehat{\Pi}^{\mathscr{C}}$ fall in a finite number of $\check{\Gamma}(S, x)^{\mathscr{C}}$-orbits which are just the closures of the $\Gamma(S, x)$-orbits of simple elements in $\Pi$. In Theorem 4.2 of [6], we showed that a procyclic subgroup of $\widehat{\Pi}^{\mathscr{C}}$ contains at most a simple element (the theorem is proved for $\mathscr{C}=\mathscr{F}$, but, as usual, it is not difficult to see that the same argument applies to any class of finite groups). We may then ask the question:

Question 7.9. Is the set of simple elements of $\widehat{\Pi}^{\mathscr{C}}$ characterized by the property that a procyclic subgroup $C$ of $\widehat{\Pi}^{\mathscr{C}}$ contains a (unique) simple element, if and only if, this subgroup is primitive (i.e. it is not properly contained in some other procyclic subgroup of $\widehat{\Pi}^{\mathscr{C}}$ ) and $\mathfrak{R}_{\mathscr{C}}(\gamma, \gamma)=0$ for some (and then all) $\gamma \in C$ ?

I think that in order to advance in all the above questions it would be useful to understand the relation of the $p$-adic Reidemeister bilinear pairing $\mathfrak{R}_{p}\left({ }_{-},-\right)$with the $p$-adic Goldman bracket introduced by Hain in [12] (cf. Section 1.2 ibid.). It would also be interesting to see whether the Goldman bracket can be defined for any class of finite groups $\mathscr{C}$ (the case $\mathscr{C}=\mathscr{F}$ would be particularly interesting). My impression is that, while the $p$-adic (or pro- $\mathscr{F}$, if any) Goldman bracket is, from a technical point of view, more difficult than the $p$-adic (or pro- $\mathscr{F}$ ) Reidemeister bilinear pairing, it also encodes more information.

\section{References}

[1] M.P. Anderson. Exactness properties of profinite completion functors. Topology 13, (1974), 229-239. 19

[2] M. Asada. The faithfulness of the monodromy representations associated with certain families of algebraic curves. Journal of Pure and Applied Algebra 159, (2001), 123-147. 4, 8, 21, 24

[3] M. Boggi. Profinite Teichmüller theory. Math. Nach. 279, n. 9-10 (2006), 953-987. 12

[4] M. Boggi. The congruence subgroup property for the hyperelliptic Teichmüller modular group: the open surface case. Hiroshima Math. J. 39 (2009), 351-362. 2, $4,22,23,25$

[5] M. Boggi. Galois covers of moduli spaces of curves and loci of curves with symmetries. Geom. Dedicata n. 168 (2014), 113-142. 12, 14 
[6] M. Boggi. On the procongruence completion of the Teichmüller modular group. Trans. Amer. Math. Soc. 366 (2014), no. 10, 5185-5221. 9, 10, 11, 12, 15, 31

[7] M. Boggi, P.A. Zalesskii. Characterizing closed curves on Riemann surfaces via homology groups of coverings. IMRN Vol. 2016, no. 00, pp. 1-22. 5, 10, 28

[8] M. Boggi, P. Zalesskii. A restricted Magnus property for profinite surface groups. To appear on "Transactions of the AMS", arXiv:1408.5419v2 (2014). 7, 9, 10, $13,14,28,29$

[9] B. Farb, D. Margalit. A Primer on Mapping Class Group. Princeton Mathematical Series 49 (2012). 3

[10] W. Fulton, J. Harris. Representation Theory: A First Course. Graduate Texts in Mathematics 129 (1991), Springer-Verlag. 26

[11] R. Hain. The de Rham homotopy theory of complex algebraic varieties, I. KTheory 1 (1987), 271-324. 30

[12] R. Hain. Hodge theory of the Goldman bracket. arXiv:1710.06053v1 (2017). 30, 31

[13] Y. Hoshi. On Monodromically Full Points of Configuration Spaces of Hyperbolic Curves. In The Arithmetic of Fundamental Groups PIA 2010. Ed. Jacob Stix. Contributions in Mathematical and Computational Sciences - Volume 2. SpringerVerlag Berlin Heidelberg (2012), 167-207. 17

[14] Y. Hoshi, Y. Ijima A pro- $\ell$ version of the congruence subgroup problem for mapping class groups of genus 1. RIMS pre-print 1791 (2014). 4

[15] Y. Hoshi, S. Mochizuki. On the combinatorial anabelian geometry of nodally nondegenerate outer representations. Hiroshima Math. J. Volume 41, Number 3 (2011), 275-342. 17

[16] E. Kani: The Galois-module structure of the space of holomorphic differentials of a curve. Journal für die reine und angewandte Mathematik 367 (1986),187-206. 26

[17] E. Looijenga. Smooth Deligne-Mumford compactifications by means of Prym level structures. J. Algebraic Geometry 3 (1994), 283-293. 15

[18] M. Matsumoto. Galois representations on profinite braid groups on curves. J. reine angew. Math. 474 (1996), 169-219. 17

[19] S. Mochizuki, A. Tamagawa. The algebraic and anabelian geometry of configuration spaces. Hokkaido Mathematical Journal Vol. 37 (2008), 75-131. 18 
[20] N. Nikolov, D. Segal. On finitely generated profinite groups, I: strong completeness and uniform bounds. Annals of Math. Vol. 165 (2006), 171-238. 5

[21] D. Puder, O. Parzanchevski. Measure preserving words are primitive. J. Amer. Math. Soc. Vol. 28, n. 1 (2015), 63-97. 5, 28

[22] L. Paris. Residual p properties of mapping class groups and surface groups. Trans. Amer. Math. Soc. 361 (2009), 2487-2507. 5, 10

[23] L. Ribes, P. Zalesskii. Profinite groups. Erg. der Math. und ihrer Grenz. 3. Folge 40, 2nd Edition, Springer-Verlag (2000). 2, 7

Marco Boggi,

Departamento de Matemática, UFMG,

Av. Antônio Carlos, 6627 - Caixa Postal 702

CEP 31270-901 - Belo Horizonte - MG, Brasil.

E-mail: marco.boggi@gmail.com 